\title{
Physiological Activity, Nutritional Composition, and Gene Expression in Apple (Malus domestica Borkh) Influenced by Different ETc Levels of Irrigation at Distinct Development Stages
}

\author{
Rafiya Mushtaq ${ }^{1, *}$, Mahinder Kumar Sharma ${ }^{1}$, Javid Iqbal Mir ${ }^{2}$, Sheikh Mansoor ${ }^{3} \mathbb{D}_{\text {, Khalid Mushtaq }}{ }^{1}$, \\ Simona Mariana Popescu ${ }^{4}$, Abdul Raouf Malik ${ }^{1}$, Hamed A. El-Serehy ${ }^{5}$, Daniel Ingo Hefft ${ }^{6}$, Sajad Ahmad Bhat ${ }^{7}$ \\ and Sumati Narayan 8
}

Citation: Mushtaq, R.; Sharma, M.K.; Mir, J.I.; Mansoor, S.; Mushtaq, K.; Popescu, S.M.; Malik, A.R.; El-Serehy, H.A.; Hefft, D.I.; Bhat, S.A.; et al. Physiological Activity, Nutritional Composition, and Gene Expression in Apple (Malus domestica Borkh) Influenced by Different ETc Levels of Irrigation at Distinct Development Stages. Water 2021, 13, 3208. https://doi.org/10.3390/w13223208

Academic Editor: Gonzalo Martínez

Received: 6 October 2021

Accepted: 30 October 2021

Published: 12 November 2021

Publisher's Note: MDPI stays neutral with regard to jurisdictional claims in published maps and institutional affiliations.

Copyright: (c) 2021 by the authors. Licensee MDPI, Basel, Switzerland. This article is an open access article distributed under the terms and conditions of the Creative Commons Attribution (CC BY) license (https:/ / creativecommons.org/licenses/by/ $4.0 /)$.
1 Division of Fruit Science, SKUAST-Kashmir, Srinagar 180009, India; drsharma_mk@rediffmail.com (M.K.S.); bhatkhalid68@gmail.com (K.M.); roufmalik@gmail.com (A.R.M.)

2 ICAR-Central Institute of Temperate Horticulture, Srinagar 190007, India; javidiqbal1234@gmail.com

3 Division of Biochemistry, SKUAST Jammu, Srinagar 180009, India; mansoorshafi21@gmail.com

4 Department of Biology and Environmental Engineering, University of Craiova, 13, A.I. Cuza, 200585 Craiova, Romania; popescu_simona83@yahoo.com

5 Department of Zoology, College of Science, King Saud University, Riyadh 11451, Saudi Arabia; hel_serehy@yahoo.com

6 Reaseheath College, University Centre Reaseheath, Nantwich CW5 6DF, UK; daniel.hefft@reaseheath.ac.uk

Division of Basic Science, SKUAST-Kashmir, Srinagar 180009, India; sajad_phy@yahoo.com

8 Division of Vegetable Science, SKUAST-Kashmir, Srinagar 180009, India; sumatinarayan@gmail.com

* Correspondence: rafiazargar.25@gmail.com

Abstract: Managing irrigation efficiently is paramount given the uncertainty in the future availability of water and rising demand for this resource. Scheduled irrigation significantly influences vegetative growth through improving crop physiology and nutrient uptake and use efficiency. Influence of different irrigation treatments (100\%, $75 \%$, and $50 \%$ volume of Class A pan evapotranspiration) applied at four different phenological stages (flowering and fruit set (C1), fruit growth stage (C2), pre-harvest stage (C3), and throughout the growing season (C4)) through drip along with a control (rainfed) on leaf physiology, nutrient content, and uptake through gene expression was studied on Super Chief Sandidge variety raised on M9T337 (5 and 6 years old) grown at a spacing of $1.5 \times 3 \mathrm{~m}$ (2222 plants/ha) under high density condition of Kashmir Himalayan range of India. A comparison of data reveals that drip irrigation at 100\% Crop evapotranspiration (ETc) increased leaf area by $60 \%$ compared to rainfed conditions. Leaf area significantly increased in plants irrigated throughout the growing season (C4) and during flowering and fruit set stage (C1). Irrigation amount likely does not have any influence on leaf development after the fruit growth stage. Stomatal opening and their size greatly vary from no irrigation to optimum irrigation in these plants. High density apple trees exposed to optimum irrigation levels (100\% and $75 \%$ ET) had significantly higher concentrations of nutrients (N, P, and $\mathrm{K}$ ) in their leaf tissues. The concentration of $\mathrm{Ca}$ and $\mathrm{Mg}$ content in leaf tissues are greatly influenced by the optimum supply of water during the early growth stages of apple growth. The availability of water significantly influences nutrient transporter gene expression and thus nutrient uptake by regulating such transporter genes. It is therefore observed that proper irrigation during $\mathrm{C} 1$ and stage $\mathrm{C} 2$ stage are the critical growth stages of apple for optimum leaf physiological activity and proper nutrient uptake.

Keywords: apple; drip-irrigation; leaf physiology; nutrient uptake; transporter genes

\section{Introduction}

Apple, Malus domestica Borkh, is the commercially grown fruit crop across globe and third-largest produced fruit in India. The cultivation of this crop has seen a drift from 
commercial cultivation to high-density plantation in recent decades. The orchardists across globe are shifting to high-density plantations due to high returns and better quality produce. However, these high-density orchards require better cultural practices and are more susceptible to water stress conditions. Such orchards cannot be irrigated with conventional flood irrigation and require scheduled irrigation through the drip. Furthermore, the current situation of water scarcity requires more efficient use of land and water resources for horticulture growth as estimates International Water Management Institute in their report, that 25 percent of the world population would experience severe water scarcity by the year 2025 [1]. Several research studies conducted worldwide show that irrigation water plays a critical role in the overall growth of crops and shows a significant influence on various tree characteristics like plant growth, rootstock function, and quality and disease-free fruit production [2,3]. In the long run, it helps in overall rural development, enhancing farmer's income through a reduction in the input cost of water, fertilizer, insecticide, herbicide, etc., and increases income through higher yields and better quality fruits [4]. Irrigation scheduling in high-density apple plantations has been reported to be the most significant factor affecting uptake of mineral nutrients, and thus tree growth [5], and as such, nutrient deficiency and water stress in these orchards are increasingly challenging their success at large scale. Scheduled irrigation is therefore a useful way to maintain a crop's physiological state and nutrient balance within the crop [6]. The researchers report that different irrigation methods had different effects on the uptake of nutrient elements [7]. Many researchers have, however, reported that any reduction in irrigation amount does not hold any significant influence on the concentration of major nutrients like $\mathrm{N}, \mathrm{P}$, and $\mathrm{K}$ in leaves [8,9]. However, it is also seen that the literature on the influence of various irrigation regimes on leaf nutrients mainly focuses on calcium $(\mathrm{Ca})$ and magnesium $(\mathrm{Mg})$ because the moment of these nutrients in plants is primarily by mass flow [10] and therefore are most affected by irrigation.

As leaves are the primary source of photosynthesis and other physiological activities, it is important to understand the influence of different levels of irrigation on these conditions. Chlorophyll content in leaves has been considered an important trait for crop production. Likewise, leaf relative water content significantly influences photosynthesis and is the appropriate measure of plant water status. As most of the water is lost by evaporation, stomas hold significant importance for monitoring plant-water balance [11], and therefore it is important to understand how irrigation levels influence stomatal density and structures in plants.

Potassium $\left(\mathrm{K}^{+}\right)$contributes to many physiological and metabolic activities, like maintenance of cellular osmolarity, neutralization of anions, and control of stomatal opening, etc., [12]. The deficiency of this nutrient significantly reduces photosynthesis resulting in poor growth and development of crops [13,14]. For optimal growth, this nutrient must be effectively absorbed by plants from the soil via roots. $\mathrm{K}^{+}$transporter genes like KT/HAK/KUP groups are ubiquitously present in plants, which implies their significant role in plant tolerance under water stress conditions $[15,16]$. However, the molecular basis of how different irrigation levels influence the expression of these genes is largely rare. Understanding the importance of these facts and the necessity to observe critical stages of irrigation depending on its influence on various crop phenological stages, which has not been addressed much in earlier studies, this work was undertaken to study the influence of different levels of irrigation on leaf physiological characteristics, nutrient concentration, and expression of transporter genes and to determine critical stages of irrigation for apple under high density plantation.

\section{Materials and Methods}

\subsection{Experimental Material}

The field experiment was executed at the experimental farm of SKUAST-K, Main campus Shalimar, J\&K, India on Super Chief Sandidge variety planted in 2013 on M9T337 dwarfing rootstock during 2017 and 2018 (Figure 1). Soil composition of the experimental farm is presented in Table 1 [17]. Irrigation was given through drip irrigation system with 
a discharge capacity of 41 /hour in one dripper during the morning hours for the whole period of the experiment. The drip line was installed $60 \mathrm{~cm}$ above ground with $40 \mathrm{~cm}$ distance between two drippers. Irrigation water used for the experiment was fresh water obtained from canal and collected in irrigation pump system. Three irrigation levels at $100 \%$ ETc, 75\% ETc, and 50\% ETc were given at various crop phenological stages along with a control (rainfed) which resulted in a total of 13 different treatment combinations (Table 2, Figure 2). The daily water requirement for each treatment was calculated using the following FAO methodology:

$$
\mathrm{ETc}=\frac{\mathrm{Epan} \times \mathrm{Kp} \times \mathrm{Kc} \times \mathrm{AA} \times \mathrm{AC}}{\mathrm{IE}}
$$

where

$\mathrm{ETc}=$ Crop evapotranspiration

Epan = Daily pan evaporation $(\mathrm{mm})$

$\mathrm{K}_{\mathrm{c}}=$ Crop coefficient

$\mathrm{K}_{\mathrm{p}}=$ Pan coefficient (taken as 0.75 )

$\mathrm{AA}=$ Area allotted per plant $\left(\mathrm{m}^{2}\right)$

$\mathrm{AC}=$ Area shaded by canopy at noon $(\%)$

IE = Irrigation efficiency of the system (90\%) taken as decimal

The total irrigation amount (liters/plant/day) that was applied was calculated using formula

$$
\text { Irrigation to be applied }((\text { liters } / \text { plant/day }))=[\text { ETc }- \text { Effective rainfall }]
$$

where Effective rainfall was calculated based on FAO guidelines

Monthly irrigation water applied at different ETc is given in Table 3.



Figure 1. View of the experimental orchard.

Table 1. Mechanical composition of soil.

\begin{tabular}{ccccc}
\hline S. No. & Particulars & Status & Textural Class & Method Followed \\
\hline 1. & Coarse sand (\%) & $0.46 \%$ & & \\
2. & Fine sand (\%) & $46.33 \%$ & Medium loam & International Pipette \\
3. & Silt (\%) & $35.00 \%$ & & \\
4. & Clay (\%) & $18.00 \%$ & & \\
\hline
\end{tabular}


Table 2. Treatment combinations used in the experiment.

\begin{tabular}{|c|c|c|c|c|}
\hline \multirow{2}{*}{ Treatment } & \multirow{2}{*}{\multicolumn{2}{|c|}{ Treatment Combination }} & \multicolumn{2}{|c|}{ Irrigation Applied (Litre/Plant) } \\
\hline & & & 2017 & 2018 \\
\hline $\mathrm{T} 1$ & Rainfed & $\mathrm{I}_{0}$ & - & - \\
\hline $\mathrm{T} 2$ & $\begin{array}{l}\text { Drip Irrigation at } 100 \% \text { ETc } \\
\text { during flowering and fruit set }\end{array}$ & $\mathrm{I}_{1} \mathrm{C}_{1}$ & 95.80 & 87.95 \\
\hline $\mathrm{T} 3$ & $\begin{array}{c}\text { Drip Irrigation at } 75 \% \text { ETc } \\
\text { during flowering and fruit set }\end{array}$ & $\mathrm{I}_{2} \mathrm{C}_{1}$ & 72.30 & 65.75 \\
\hline $\mathrm{T} 4$ & $\begin{array}{l}\text { Drip Irrigation at } 50 \% \text { ETc } \\
\text { during flowering and fruit set }\end{array}$ & $\mathrm{I}_{3} \mathrm{C}_{1}$ & 47.60 & 43.78 \\
\hline T5 & $\begin{array}{l}\text { Drip Irrigation at } 100 \% \text { ETc } \\
\text { during fruit growth stage }\end{array}$ & $\mathrm{I}_{1} \mathrm{C}_{2}$ & 320.61 & 350.25 \\
\hline T6 & $\begin{array}{l}\text { Drip Irrigation at } 75 \% \text { ETc } \\
\text { during fruit growth stage }\end{array}$ & $\mathrm{I}_{2} \mathrm{C}_{2}$ & 240.08 & 262.10 \\
\hline $\mathrm{T} 7$ & $\begin{array}{l}\text { Drip Irrigation at } 50 \% \text { ETc } \\
\text { during fruit growth stage }\end{array}$ & $\mathrm{I}_{3} \mathrm{C}_{2}$ & 158.90 & 174.36 \\
\hline $\mathrm{T} 8$ & $\begin{array}{l}\text { Drip Irrigation at } 100 \% \text { ETc } \\
\text { during pre-harvest stage }\end{array}$ & $\mathrm{I}_{1} \mathrm{C}_{3}$ & 214.35 & 182.18 \\
\hline T9 & $\begin{array}{l}\text { Drip Irrigation at } 75 \% \text { ETc } \\
\text { during pre-harvest stage }\end{array}$ & $\mathrm{I}_{2} \mathrm{C}_{3}$ & 160.78 & 136.23 \\
\hline $\mathrm{T} 10$ & $\begin{array}{l}\text { Drip Irrigation at } 50 \% \text { ETc } \\
\text { during pre-harvest stage }\end{array}$ & $\mathrm{I}_{3} \mathrm{C}_{3}$ & 106.80 & 89.90 \\
\hline $\mathrm{T} 11$ & $\begin{array}{l}\text { Drip Irrigation at } 100 \% \text { ETc } \\
\text { throughout the growing season }\end{array}$ & $\mathrm{I}_{1} \mathrm{C}_{4}$ & 631.30 & 638.20 \\
\hline $\mathrm{T} 12$ & $\begin{array}{l}\text { Drip Irrigation at } 75 \% \text { ETc } \\
\text { throughout the growing season }\end{array}$ & $\mathrm{I}_{2} \mathrm{C}_{4}$ & 472.80 & 464.00 \\
\hline $\mathrm{T} 13$ & $\begin{array}{l}\text { Drip Irrigation at } 50 \% \text { ETc } \\
\text { throughout the growing season }\end{array}$ & $\mathrm{I}_{3} \mathrm{C}_{4}$ & 306.70 & 307.90 \\
\hline
\end{tabular}
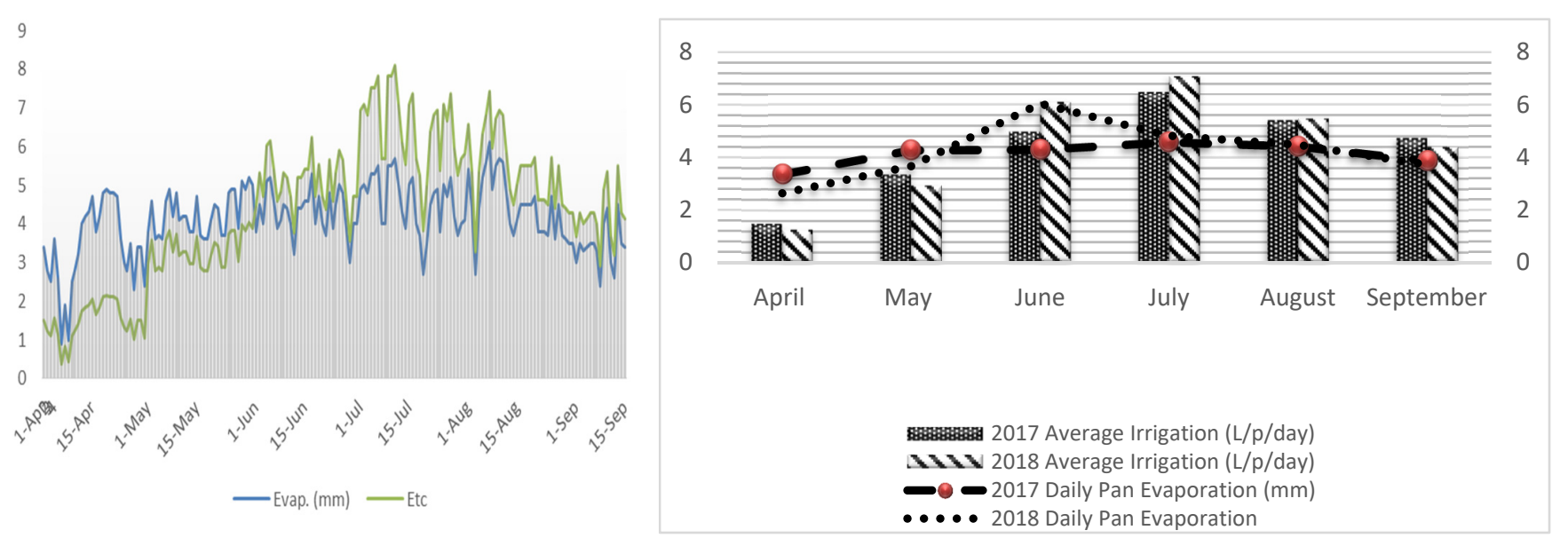

Figure 2. Average daily irrigation requirement (liter/plant/day) and pan evaporation for apple during the growing period.

\subsection{Location and Weather}

The experimental farms of the SKUAST-K campus are at an elevation of $1605 \mathrm{~m}$ above mean sea level with latitude and longitude of $34^{\circ} 05^{\prime} \mathrm{N}$ and $74^{\circ} 50^{\prime} \mathrm{E}$, respectively. During winter the average day temperature is $\sim 2.5^{\circ} \mathrm{C}$, and night temperatures are below freezing. Summers are usually warm. The annual rainfall during 2017 and 2018 was 1229.9 and $790.04 \mathrm{~mm}$, respectively, with annual evaporation of $998.98 \mathrm{~mm}$ and $1798.9 \mathrm{~mm}$ during the respective years. The maximum and minimum temperature and relative humidity during the experimental period are shown in Figure 3. The daily and monthly weather data and pan evaporation data were recorded from the Agrometrological station of SKUAST-K, Shalimar, Srinagar, during experimentation. 


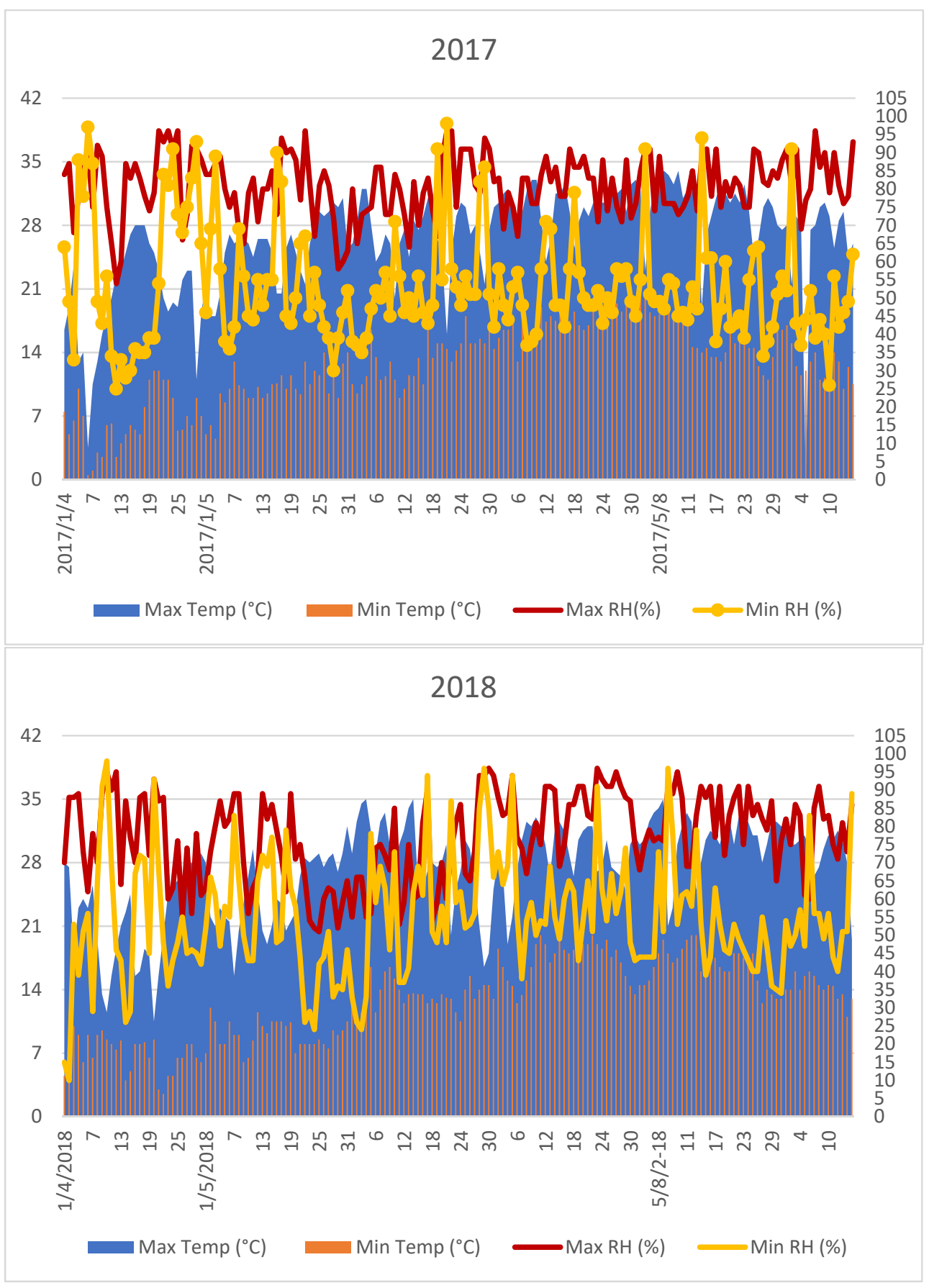

Figure 3. Daily meteorological data during experimental period (2017 and 2018).

\subsection{Leaf Physiological Characteristics}

The leaf area of each sample comprising of 30 leaves was collected at random from different directions of each experimental tree and measured with the help of a leaf area meter (221 Systronics) and expressed in square centimeters. Furthermore, leaf area at the end of $C_{1}$ stage, $C_{2}$ stage, and harvest were recorded to observe the change in leaf area during different growth stages. Chlorophyll content in leaf samples (mg/g fresh weight) was estimated by Dimethyl sulfoxide (DMSO) method given by Arnon [18]. Chlorophyll 
content was calculated using the formula given by Richardson et al. [19] and expressed in terms of $\mathrm{mg} / \mathrm{g}$ fresh weight.

$$
\text { Total chlorophyll }(\mathrm{a}+\mathrm{b})=\frac{20.2(\mathrm{~A} 645)+8.02(\mathrm{~A} 663) \times \mathrm{V}}{1000 \times \mathrm{W}}
$$

$\mathrm{W}=$ Weight of plant tissue $(\mathrm{g})$

$\mathrm{A}=$ Absorbance at specific wavelength (645 and 663)

$\mathrm{V}=$ final volume of DMSO $(\mathrm{mL})$

The leaf relative water content was determined as described by Slavik [20]. The relative water content (RWC) was then calculated using the formula:

$$
\text { RWC }=\frac{\text { Fresh Weight }(\text { FW })-\text { Dry Weight }(\text { DW })}{\text { Turgid Weight }(\text { TW })-\text { Dry Weight }(\text { DW })} \times 100
$$

Stomatal studies. The number of stomata in the leaves of treated plants was measured with the help of ocular and stage micrometer (ERMA, Tokyo, Japan). A thin layer of a quick fix was applied on the undersurface of tagged leaves. After drying the peels were separated and put on slides to be observed under the microscope as suggested by Beakbane and Majumdar [21] using $4 \times \times 100 \times$ magnification (Figure 4).

$$
\text { Number of stomata per microscopic field }=\frac{\text { Stomata number } / \mathrm{mm}^{2}}{\text { Area of microscopic field }\left(\mathrm{mm}^{2}\right)}
$$

\begin{tabular}{|c|c|c|c|c|}
\hline \multirow{2}{*}{ Month } & \multirow{2}{*}{ ETc } & \multicolumn{3}{|c|}{ Total Irrigation (Liter) 2017} \\
\hline & & $100 \%$ ETc & $75 \%$ ETc & $50 \%$ ETc \\
\hline April & 44.2 & 17.4 & 13.2 & 8.6 \\
\hline May & 103.2 & 78.4 & 59.1 & 32.4 \\
\hline June & 152.0 & 133.8 & 100.3 & 66.1 \\
\hline July & 201.2 & 186.8 & 139.7 & 92.8 \\
\hline August & 167.0 & 159.4 & 119.1 & 79.5 \\
\hline September & 62.5 & 55.5 & 41.4 & 27.3 \\
\hline Total & 730.3 & 631.3 & 472.8 & 306.7 \\
\hline \multirow{2}{*}{ Month } & \multirow{2}{*}{ ETc } & \multicolumn{3}{|c|}{ Total Irrigation (Liter) 2018} \\
\hline & & $100 \%$ ETc & $75 \%$ ETc & $50 \%$ ETc \\
\hline April & 37.6 & 13.4 & 10 & 6.7 \\
\hline May & 90.8 & 74.5 & 55.7 & 37 \\
\hline June & 181.3 & 170.5 & 127.6 & 84.8 \\
\hline July & 219.6 & 197.7 & 134.5 & 89.5 \\
\hline August & 169.0 & 109.7 & 82.3 & 54.1 \\
\hline September & 72.6 & 72.4 & 53.9 & 35.8 \\
\hline Total & 770.9 & 638.2 & 464.0 & 307.9 \\
\hline
\end{tabular}

Table 3. Monthly water requirement at three ETc levels (100\%, $75 \%$, and $50 \%$ ) for high density apple plantation (2017 and 2018).

\subsection{Leaf Nutrient Status}

Fresh leaf samples from all the treatments were collected and processed for analysis in mid-august as per the protocol given by [22]. Processed leaf samples were digested for nitrogen estimation following protocol as suggested by Jackson [23]. Digestion of leaf samples for $\mathrm{P}, \mathrm{K}, \mathrm{Ca}$, and $\mathrm{Mg}$ nutrient estimation was done using diacid mixture of nitric acid and perchloric acid in the ratio of 4:1. 


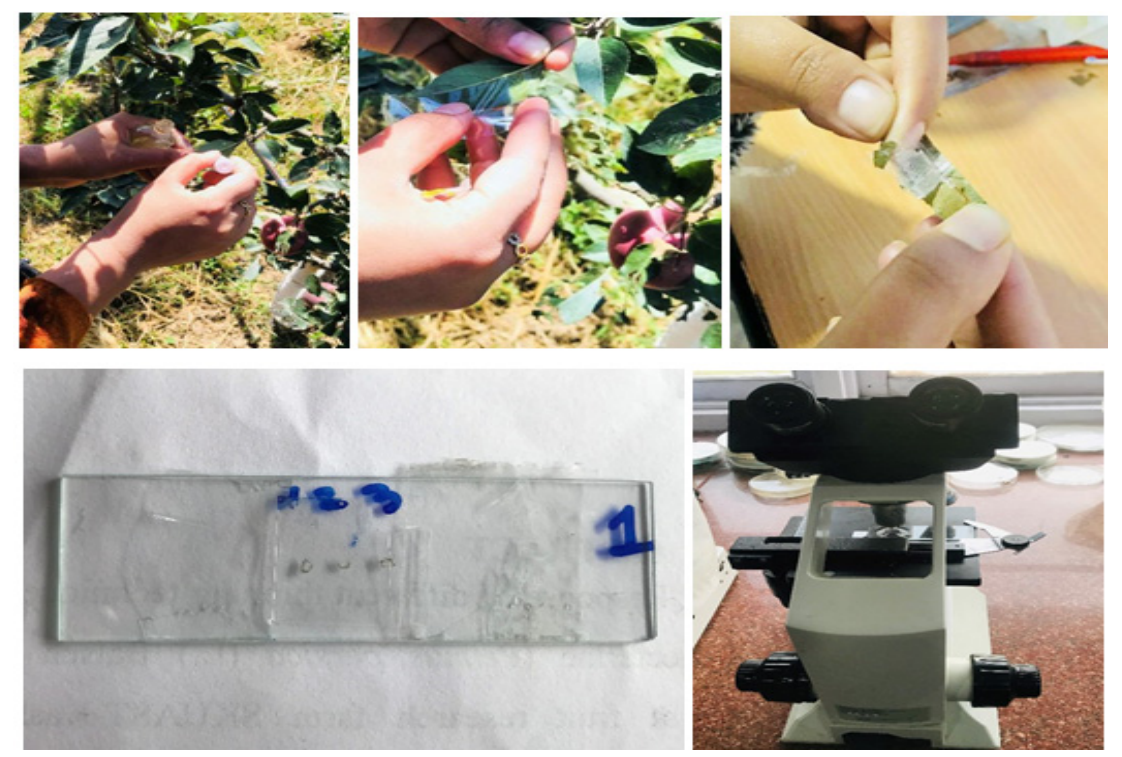

Figure 4. Stomatal studies using ocular and stage micrometer.

\subsection{Gene Expression Studies}

\subsubsection{RNA Extraction and cDNA Synthesis}

For RNA extraction, the leaves collected from various treatments were immediately dipped in liquid nitrogen and frozen leaves were ground in sterilized mortar and pestle into a fine powder using liquid nitrogen and total RNA was extracted using Trizol Reagent (Sigma Aldrich, St. Louis, MO, USA) following the manufacturer's protocol. All the material used was properly treated with diethyl pyrocarbonate (DEPC-RNase inhibitor) to completely prevent RNase contamination. The RNA was quantified using NanoDrop 1000 (Thermo Fisher, Waltham, MA, USA) at absorbance 260 and $280 \mathrm{~nm}$, and RNA with a ratio of 1.5 to 2.0 OD was used for comparative studies.

Complementary DNA (cDNA) was generated using oligodT primers for first-strand cDNA synthesis using as described by the manufacturer (RevertAid First Strand cDNA Synthesis Kit). The synthesized cDNA was stored at $-20{ }^{\circ} \mathrm{C}$ for gene expression study.

\subsubsection{RT-qPCR Analysis}

Reverse transcription was carried out for amplification of MdNFPr and POTT gene using tubulin gene as an internal control. RNA concentration @ $1000 \mu \mathrm{g}(1 \mathrm{mg})$ was used for each reaction to equilibrate the total reactions for gene expression. An RNase-free environment was created for the handling of RNA. Forward and reverse primers sequences are presented in the Table 4. PCR reactions were performed in a thermocycler (Takara, Maebashi, Japan) with 2-5 $\mu$ g of cDNA. The annealing temperature of all the primers was optimized using gradient PCR. PCR products (5 $\mu \mathrm{L}$ each) were run on $1.2 \%(w / v)$ agarose gel (Sigma-Aldrich, Burlington, MA, USA).

Table 4. Primer sequences for amplification of nutrient transporter genes in apple with expected amplicons lengths.

\begin{tabular}{|c|c|c|c|c|}
\hline Primer & Description & Forward Primer $\left(5^{\prime}-3^{\prime}\right)$ & Reverse Primer $3^{\prime}-5^{\prime}$ & Amplicon Size (bp) \\
\hline MdNFPr & $\begin{array}{c}\text { Nitrogen } \\
\text { transporter gene }\end{array}$ & GGCCAACCAGGTTTCACTAA & ACCCACCAGGAAGAACTGTG & 170 \\
\hline POTT & $\begin{array}{l}\text { Potassium } \\
\text { transporter gene }\end{array}$ & CAGAGTTCATTCGGTCAGA & CTTGCTTCCATCAGTTCCT & 277 \\
\hline
\end{tabular}

Real-time PCR was performed in a LightCycler 480 real-time PCR instrument (Roche Diagnostics (Basel, Switzerland)) using the SYBR Green I Master kit (LightCycler 480). Reactions were performed in triplicate and contained $5 \mu \mathrm{L}$ SYBR Green I Master, $2 \mu \mathrm{L}$ PCR-grade water, $1.5 \mu \mathrm{L} c \mathrm{cNA}$, and $0.5 \mu \mathrm{L}$ of each of the $10 \mu \mathrm{M}$ forward and reverse gene- 
specific primers in a final volume of $10 \mu \mathrm{L}$. Tubulin gene was taken as a reference gene and POTT gene expression during drip irrigation at 100\% ETc throughout the growing season as a positive control. Fluorescence data was collected using LightCycler 480 software (version 1.5; Roche Diagnostics) [24].

\subsection{Statistical Analysis}

The results obtained were subjected to one-way analysis of variance (ANOVA) and Duncan's Multiple range test. All statistical tests were done using SAS software.

\section{Results}

\subsection{Leaf Physiological Characteristics}

The highest leaf area of $25.60 \mathrm{~cm}^{2}$ was recorded in $100 \%$ ETc which was 24 percent higher in comparison to rainfed conditions (Table 5, Figure 5). Similarly, plants irrigated throughout the growing season $\left(C_{4}\right)$ registered the highest leaf area $\left(30.68 \mathrm{~cm}^{2}\right)$. The leaf area under different interactions ranged between $19.85 \mathrm{~cm}^{2}$ to $31.85 \mathrm{~cm}^{2}$. The highest leaf area was observed in $\mathrm{I}_{1} \mathrm{C}_{4}$ interaction $\left(31.85 \mathrm{~cm}^{2}\right)$ which was 40 percent higher compared to rainfed conditions followed by $\mathrm{I}_{2} \mathrm{C}_{4}\left(31.06 \mathrm{~cm}^{2}\right)$ (Figure 5). Leaf chlorophyll content varied between 1.53 and $2.23 \mathrm{mg} / \mathrm{g}$ FW based on two years pooled data under present investigation and was significantly higher under different levels of irrigation compared to rainfed conditions (Table 5; Figure 6). The leaf chlorophyll content was noted to be $\sim 21$ percent higher under $\mathrm{I}_{1}$ level of irrigation $(100 \%$ ETc) as compared to rainfed plants.

Table 5. Effect of different irrigation levels at various crop phenological stages on leaf area and leaf chlorophyll content (mg/g fresh weight) of apple (cv. Super Chief Sandidge).

\begin{tabular}{|c|c|c|c|c|c|c|}
\hline \multirow{2}{*}{$\begin{array}{l}\text { Irrigation } \\
\text { Level (I) }\end{array}$} & \multicolumn{3}{|c|}{ Leaf Area $\left(\mathrm{cm}^{2}\right)$} & \multicolumn{3}{|c|}{ Leaf Chlorophyll (mg/g) } \\
\hline & 2017 & 2018 & Pooled & 2017 & 2018 & Pooled \\
\hline $\mathrm{I}_{0}$ & $19.12^{b}$ & $19.58^{c}$ & $18.85^{\mathrm{c}}$ & $1.59^{b}$ & $1.48^{\mathrm{b}}$ & $1.53^{\mathrm{b}}$ \\
\hline $\mathrm{I}_{1}$ & $25.45^{a}$ & $25.75^{a}$ & $25.60^{\mathrm{a}}$ & $2.00^{\mathrm{a}}$ & $1.96^{\mathrm{a}}$ & $1.96^{\mathrm{a}}$ \\
\hline $\mathrm{I}_{2}$ & $25.02^{a}$ & $25.16^{\mathrm{a}}$ & $25.09^{a}$ & $1.97^{\mathrm{a}}$ & $1.94^{\mathrm{a}}$ & $1.94^{\mathrm{a}}$ \\
\hline $\mathrm{I}_{3}$ & $23.78^{b}$ & $22.37^{b}$ & $23.57^{b}$ & $1.95^{\mathrm{a}}$ & $1.85^{\mathrm{ab}}$ & $1.90^{\mathrm{a}}$ \\
\hline $\operatorname{Pr}>\mathrm{F}$ & 0.0182 & 0.0184 & 0.013 & 0.041 & 0.002 & $<0.0001$ \\
\hline \multicolumn{7}{|c|}{ Crop Phenological Stage (C) } \\
\hline $\mathrm{C}_{1}$ & $23.67^{b}$ & $26.83^{b}$ & $24.99^{b}$ & $1.74^{\mathrm{b}}$ & $1.71^{\mathrm{c}}$ & $1.71^{\mathrm{c}}$ \\
\hline $\mathrm{C}_{2}$ & $23.15^{b}$ & $22.03^{c}$ & $23.03^{c}$ & $1.97 \mathrm{ab}$ & $1.93^{b}$ & $1.95^{b}$ \\
\hline $\mathrm{C}_{3}$ & $21.00^{c}$ & $20.30^{\mathrm{d}}$ & $20.65^{d}$ & $1.97 \mathrm{ab}$ & $1.91^{b}$ & $1.94^{\mathrm{b}}$ \\
\hline $\mathrm{C}_{4}$ & $30.52^{\mathrm{a}}$ & $30.84^{\mathrm{a}}$ & $30.68^{a}$ & $2.21^{\mathrm{a}}$ & $2.10^{\mathrm{a}}$ & $2.16^{\mathrm{a}}$ \\
\hline $\operatorname{Pr}>\mathrm{F}$ & $<0.0001$ & $<0.0001$ & $<0.0001$ & 0.035 & 0.002 & $<0.0001$ \\
\hline \multicolumn{7}{|c|}{ Irrigation Level $\times$ Crop Phenological Stage $(\mathrm{IC})$} \\
\hline $\mathrm{I}_{0}$ & $19.12^{\mathrm{i}}$ & $19.58^{\mathrm{h}}$ & $18.85 \mathrm{~g}$ & $1.59^{\mathrm{c}}$ & $1.48^{\mathrm{e}}$ & $1.53^{\mathrm{d}}$ \\
\hline $\mathrm{I}_{1} \mathrm{C}_{1}$ & $25.64^{\mathrm{d}}$ & $27.40^{c}$ & $26.04^{\mathrm{c}}$ & $1.78^{a b c}$ & $1.74 \mathrm{bcd}$ & $1.76^{\mathrm{cd}}$ \\
\hline $\mathrm{I}_{2} \mathrm{C}_{1}$ & 23.84 ef & $26.95^{\mathrm{cd}}$ & $25.19^{\mathrm{cd}}$ & $1.73^{\mathrm{abc}}$ & $1.72 \mathrm{~cd}$ & $1.72^{\mathrm{cd}}$ \\
\hline $\mathrm{I}_{3} \mathrm{C}_{1}$ & 20.61 hi & $26.14^{\mathrm{d}}$ & 23.73 de & $1.71 \mathrm{bc}$ & $1.69^{\mathrm{d}}$ & $1.70^{\mathrm{cd}}$ \\
\hline $\mathrm{I}_{1} \mathrm{C}_{2}$ & 24.68 de & $23.14^{\mathrm{e}}$ & 24.39 cde & $1.98^{a b c}$ & $1.94^{\mathrm{bc}}$ & $1.96^{\mathrm{ab}}$ \\
\hline $\mathrm{I}_{2} \mathrm{C}_{2}$ & $23.44^{\mathrm{f}}$ & $22.08^{f}$ & $22.96^{\mathrm{ef}}$ & $1.97 \mathrm{abc}$ & $1.93 \mathrm{bc}$ & $1.95^{\mathrm{ab}}$ \\
\hline $\mathrm{I}_{3} \mathrm{C}_{2}$ & $21.33 \mathrm{gh}$ & $21.99^{f}$ & $21.30 \mathrm{fg}$ & $1.97 \mathrm{abc}$ & $1.91 \mathrm{bc}$ & $1.94^{\mathrm{ab}}$ \\
\hline $\mathrm{I}_{1} \mathrm{C}_{3}$ & 20.88 ghi & $20.75 \mathrm{~g}$ & $21.82 \mathrm{~g}$ & $1.96^{\mathrm{abc}}$ & $1.82^{b c d}$ & $1.90^{b c}$ \\
\hline $\mathrm{I}_{2} \mathrm{C}_{3}$ & $21.86^{g}$ & $20.19 \mathrm{gh}$ & $20.02^{g}$ & $1.99 \mathrm{abc}$ & $1.97^{\mathrm{b}}$ & $1.97^{\mathrm{c}}$ \\
\hline $\mathrm{I}_{3} \mathrm{C}_{3}$ & $20.27 \mathrm{hi}$ & $19.96 \mathrm{gh}$ & $20.11^{\mathrm{g}}$ & $1.97 \mathrm{abc}$ & $1.95^{b c}$ & $1.96^{\mathrm{c}}$ \\
\hline $\mathrm{I}_{1} \mathrm{C}_{4}$ & $31.96^{\mathrm{a}}$ & $31.70^{\mathrm{a}}$ & $31.85^{\mathrm{a}}$ & $2.26^{\mathrm{a}}$ & $2.22^{\mathrm{a}}$ & $2.23^{a}$ \\
\hline $\mathrm{I}_{2} \mathrm{C}_{4}$ & $30.70^{b}$ & $31.43^{\mathrm{a}}$ & $31.06^{\mathrm{a}}$ & $2.21^{\mathrm{ab}}$ & $2.19^{a}$ & $2.18^{a}$ \\
\hline $\mathrm{I}_{3} \mathrm{C}_{4}$ & $28.91^{\mathrm{C}}$ & $29.40^{b}$ & $29.15^{b}$ & $1.97 \mathrm{abc}$ & $1.99^{b}$ & $1.98^{a b}$ \\
\hline $\operatorname{Pr}>F$ & $<0.0001$ & $<0.0001$ & $<0.0001$ & 0.008 & $<0.0001$ & $<0.0001$ \\
\hline
\end{tabular}

Means followed by the same letter within the columns are not significantly different $(p=0.05)$ using Duncan's multiple range test. Means followed by the same letter within the columns are not significantly different $(p=0.05)$ using Duncan's multiple range test. I0-No irrigation, I1-100\% ETc, I2-75\% ETc, I3-50\% ETc; C1-Flowering and fruit set (April-May), C2-Fruit growth stage (June-July), C3-Pre-harvest stage (August-15 September), C4-throughout the growing season (April-September). 


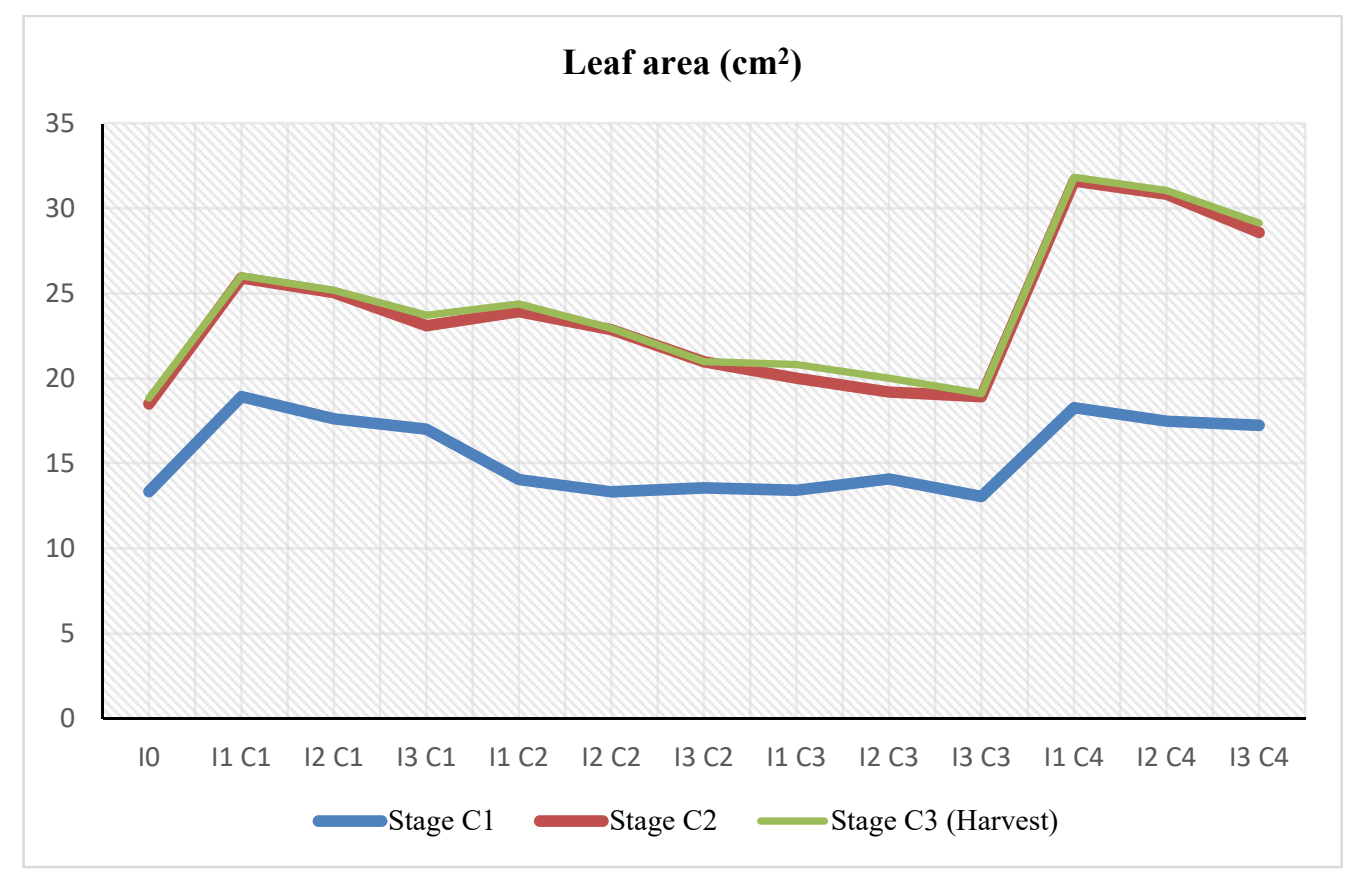

Figure 5. Change in leaf area during various crop phenological stages in response to different levels of irrigation.

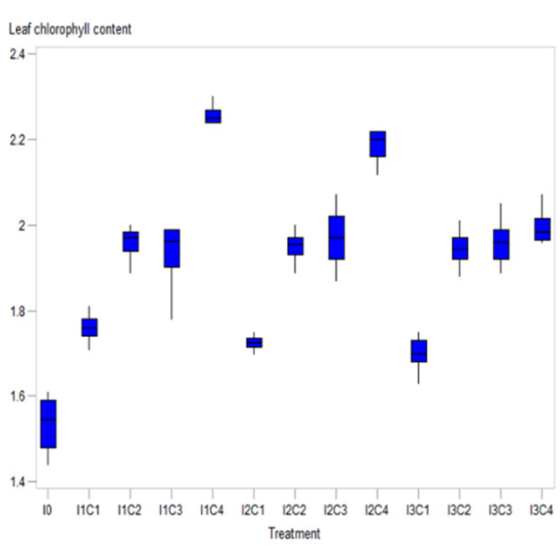

(a)

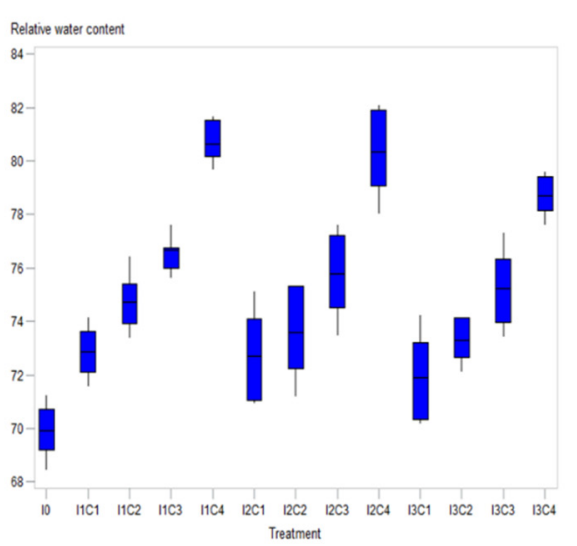

(b)

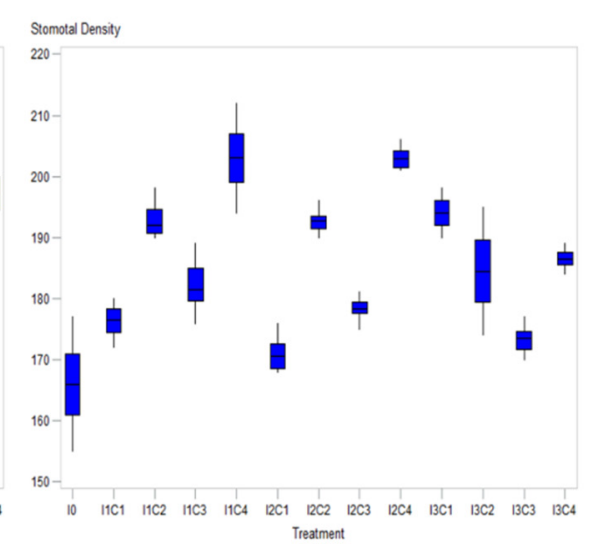

(c)

Figure 6. Influence of different irrigation levels at various crop phenological stages on (a) leaf chlorophyll content, (b) relative water content, and (c) stomatal density.

The results of the present study revealed that leaf relative water content ranged between 69.91 to 81.04 percent during the two years of study (Table 6; Figure 6). The highest leaf relative water content $(76.29 \%$ ) was noticed in plants irrigated at $100 \%$ ETc which significantly decreased with lower levels of ETc and was found lowest under rainfed conditions. Plants irrigated throughout the growing season $\left(\mathrm{C}_{4}\right.$ stage $)$ registered the highest leaf relative water content followed by plants irrigated during the fruit growth stage. Further, it was observed from the interaction effect that increases in leaf relative water content varied in the range of 3 to 9 percent as compared to rainfed conditions. Leaf relative water content decreased by 16 percent under rainfed conditions when compared to leaf relative water content from plants irrigated at $100 \%$ ETc throughout the growing season. 
Table 6. Effect of different irrigation levels at various crop phenological stages on leaf relative water content and stomatal density of apple (cv. Super Chief Sandidge).

\begin{tabular}{|c|c|c|c|c|c|c|}
\hline \multirow{2}{*}{$\begin{array}{c}\text { Irrigation } \\
\text { Level (I) }\end{array}$} & \multicolumn{3}{|c|}{ Leaf Relative Water Content (\%) } & \multicolumn{3}{|c|}{ Stomatal Density $(\mathrm{mm})$} \\
\hline & 2017 & 2018 & Pooled & 2017 & 2018 & Pooled \\
\hline $\mathrm{I}_{0}$ & $70.20^{c}$ & $69.63^{c}$ & $69.91^{c}$ & $167.00^{\mathrm{b}}$ & $165.00^{\mathrm{b}}$ & $166.20^{c}$ \\
\hline $\mathrm{I}_{1}$ & $76.14^{\mathrm{a}}$ & $76.44^{\mathrm{a}}$ & $76.29^{a}$ & $188.00^{\mathrm{a}}$ & $191.00^{\mathrm{a}}$ & $189.50^{\mathrm{a}}$ \\
\hline $\mathrm{I}_{2}$ & $75.48^{a b}$ & $75.73^{a b}$ & $75.60^{a b}$ & $187.50^{\mathrm{a}}$ & $186.50^{\mathrm{a}}$ & $187.12^{a}$ \\
\hline $\mathrm{I}_{3}$ & $74.84^{\mathrm{b}}$ & $74.78^{b}$ & $74.79^{b}$ & $179.00 \mathrm{ab}$ & $177.50 \mathrm{ab}$ & $178.75^{b}$ \\
\hline $\operatorname{Pr}>F$ & 0.038 & 0.032 & 0.0002 & 0.036 & 0.039 & 0.0003 \\
\hline \multicolumn{7}{|c|}{ Crop Phenological Stage (C) } \\
\hline $\mathrm{C}_{1}$ & $72.80^{c}$ & $72.20^{c}$ & $72.50^{\mathrm{d}}$ & 174.66 & 173.00 & $173.83^{b}$ \\
\hline $\mathrm{C}_{2}$ & $73.60^{c}$ & $74.16^{\mathrm{b}}$ & $73.88^{c}$ & 190.33 & 190.66 & $190.50^{\mathrm{a}}$ \\
\hline $\mathrm{C}_{3}$ & $75.76^{b}$ & $75.19^{b}$ & $75.83^{b}$ & 178.00 & 177.00 & $177.50^{b}$ \\
\hline $\mathrm{C}_{4}$ & $79.78^{a}$ & $80.28^{a}$ & $80.03^{a}$ & 196.66 & 197.00 & $196.83^{a}$ \\
\hline $\operatorname{Pr}>\mathrm{F}$ & $<0.0001$ & $<0.0001$ & $<0.0001$ & (NS) & (NS) & 0.0002 \\
\hline \multicolumn{7}{|c|}{ Irrigation Level $\times$ Crop Phenological Stage $(\mathrm{IC})$} \\
\hline $\mathrm{I}_{0}$ & $70.20 \mathrm{~g}$ & $69.63^{\mathrm{h}}$ & $69.91^{\mathrm{h}}$ & $167.00^{\mathrm{d}}$ & $165.00^{\mathrm{d}}$ & $166.20^{\mathrm{e}}$ \\
\hline $\mathrm{I}_{1} \mathrm{C}_{1}$ & 73.13 ef & $72.62 \mathrm{~g}$ & $72.87 \mathrm{fg}$ & $176.00^{\mathrm{c}}$ & $175.00^{\mathrm{c}}$ & $176.50^{\mathrm{cd}}$ \\
\hline $\mathrm{I}_{2} \mathrm{C}_{1}$ & 73.07 ef & $72.36^{\mathrm{g}}$ & $72.71 \mathrm{fg}$ & $177.00^{\mathrm{c}}$ & $175.00^{\mathrm{c}}$ & $176.00^{\mathrm{cd}}$ \\
\hline $\mathrm{I}_{3} \mathrm{C}_{1}$ & $72.21^{\mathrm{f}}$ & $71.62^{\mathrm{gh}}$ & $71.91^{\mathrm{g}}$ & $171.00^{\mathrm{cd}}$ & $169.00^{\mathrm{cd}}$ & $170.00^{d}$ \\
\hline $\mathrm{I}_{1} \mathrm{C}_{2}$ & $74.41^{\mathrm{de}}$ & $75.08 \mathrm{~d}^{\text {ef }}$ & 74.74 de & $194.00^{a b}$ & $195.00^{a b}$ & $194.50^{\mathrm{ab}}$ \\
\hline $\mathrm{I}_{2} \mathrm{C}_{2}$ & $73.25^{\mathrm{ef}}$ & 73.93 efg & 73.59 ef & $193.00^{a b}$ & $192.00^{\mathrm{b}}$ & $192.50^{b}$ \\
\hline $\mathrm{I}_{3} \mathrm{C}_{2}$ & 73.14 ef & $73.47 \mathrm{gf}$ & $73.30 \mathrm{fg}$ & $184.00 \mathrm{bc}$ & $185.00^{b c}$ & $184.50 \mathrm{bc}$ \\
\hline $\mathrm{I}_{1} \mathrm{C}_{3}$ & $76.36^{c}$ & $76.60^{\mathrm{cd}}$ & $76.51^{\mathrm{c}}$ & $180.00^{b c}$ & $179.00^{b c d}$ & $182.30^{b c}$ \\
\hline $\mathrm{I}_{2} \mathrm{C}_{3}$ & $75.54^{\mathrm{cd}}$ & 75.99 de & $75.76^{\mathrm{cd}}$ & $180.00 \mathrm{bc}$ & $178.00 \mathrm{bcd}$ & $179.00^{\mathrm{cd}}$ \\
\hline $\mathrm{I}_{3} \mathrm{C}_{3}$ & $75.39 \mathrm{~cd}$ & $75.09 \mathrm{~d}^{\text {ef }}$ & $75.24^{\mathrm{cd}}$ & $174.00^{\mathrm{cd}}$ & $174.00^{\mathrm{c}}$ & $174.00^{\mathrm{cd}}$ \\
\hline $\mathrm{I}_{1} \mathrm{C}_{4}$ & $80.67^{a}$ & $81.41^{\mathrm{a}}$ & $81.04^{a}$ & $202.00^{\mathrm{a}}$ & $204.00^{a}$ & $203.00^{a}$ \\
\hline $\mathrm{I}_{2} \mathrm{C}_{4}$ & $80.07^{a b}$ & $80.63^{a b}$ & $80.35^{a}$ & $201.00^{a}$ & $201.00^{a b}$ & $201.00^{a b}$ \\
\hline $\mathrm{I}_{3} \mathrm{C}_{4}$ & $78.62^{b}$ & $78.81^{b c}$ & $78.71^{\mathrm{b}}$ & $187.00^{\mathrm{b}}$ & $186.00^{b c}$ & $186.50^{b c}$ \\
\hline $\operatorname{Pr}>F$ & $<0.0001$ & $<0.0001$ & $<0.0001$ & 0.045 & 0.008 & 0.016 \\
\hline
\end{tabular}

The pooled two-year data indicated that stomatal density ranged between 166.20 to $203 \mathrm{~mm}^{-2}$ in response to different levels of irrigation at various crop phenological stages (Table 6; Figures 6 and 7). Stomatal density recorded in leaves from plants irrigated at $100 \%$ ETc was 12.2 percent higher compared to control (rainfed conditions). Similarly, stomatal density was observed highest in leaves from plants irrigated throughout the growing season which had no statistical significance with trees irrigated during the fruit growth stage. Among various combinations, $\mathrm{I}_{1} \mathrm{C}_{4}$ showed the highest stomatal density of $203.00 \mathrm{~mm}^{-2}$ which was 18 percent higher than rainfed conditions, and an increase in stomatal density under various combinations ranged between 2 to 18 percent compared to rainfed conditions. Similarly, significant variations were observed with respect to stomatal size. Plants irrigated at $100 \%$ ETc registered the highest stomatal size $(37.8 \times 29.4 \mu \mathrm{m})$, followed by plants irrigated at lower levels of ETc while the lowest stomata size was observed in plants from rainfed conditions $(21.0 \times 16.8 \mu \mathrm{m})$ (Table 7). 


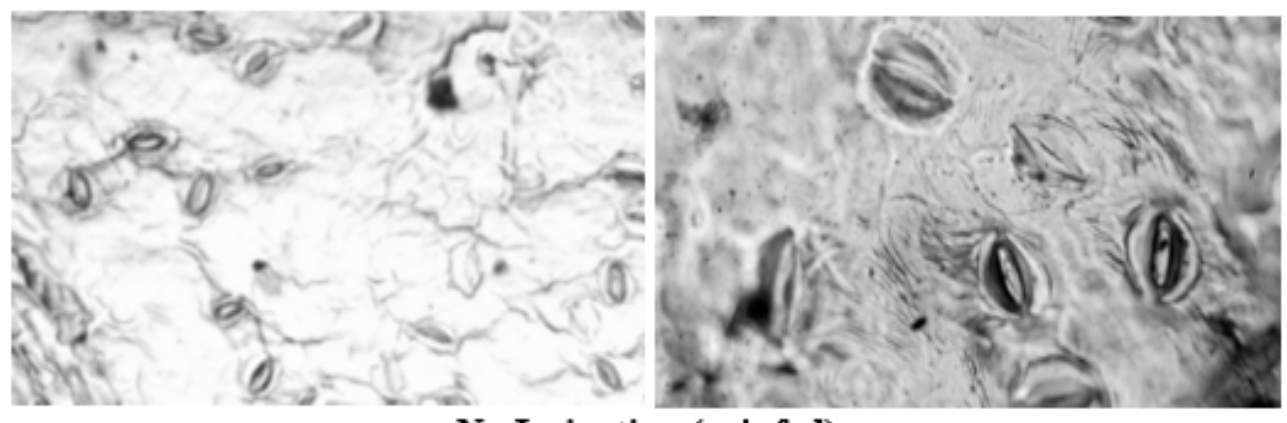

No Irrigation (rainfed)

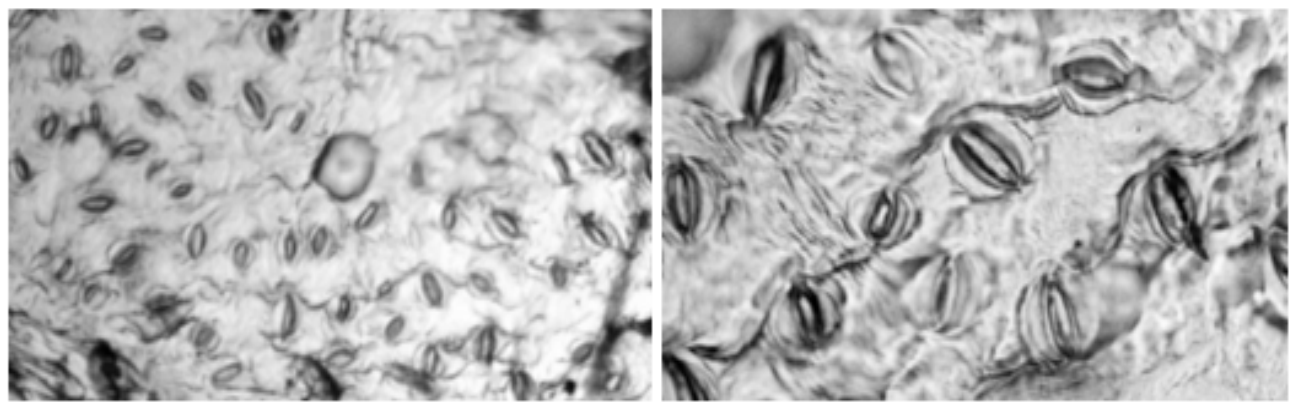

Full Irrigation $\left(\mathrm{I}_{1} \mathrm{C}_{4}\right)$

Figure 7. Stomatal density (No. of stomata $/ \mathrm{mm}^{2}$ ) under different levels of irrigation.

Table 7. Effect of different irrigation levels at various crop phenological stages on stomatal size ( $\mu \mathrm{m})$ of apple (cv. Super Chief Sandidge).

\begin{tabular}{ccc}
\hline \multirow{2}{*}{ Treatment } & \multicolumn{2}{c}{ Length (L) $\times$ Width (W) } \\
\cline { 2 - 3 } & 2017 & 2018 \\
\hline $\mathrm{I}_{0}$ & $25.2^{\mathrm{f}} \times 16.8^{\mathrm{de}}$ & $21.0^{\mathrm{g}} \times 16.8^{\mathrm{f}}$ \\
\hline $\mathrm{I}_{1} \mathrm{C}_{1}$ & $29.4^{\mathrm{e}} \times 16.6^{\mathrm{de}}$ & $29.4^{\mathrm{e}} \times 17.8^{\mathrm{f}}$ \\
\hline $\mathrm{I}_{2} \mathrm{C}_{1}$ & $28.4^{\mathrm{e}} \times 16.7^{\mathrm{e}}$ & $28.2^{\mathrm{e}} \times 16.8^{\mathrm{f}}$ \\
\hline $\mathrm{I}_{3} \mathrm{C}_{1}$ & $33.6^{\mathrm{c}} \times 16.8^{\mathrm{e}}$ & $25.2^{\mathrm{f}} \times 21.00^{\mathrm{c}}$ \\
\hline $\mathrm{I}_{1} \mathrm{C}_{2}$ & $32.8^{\mathrm{c}} \times 19.8^{\mathrm{e}}$ & $33.8^{\mathrm{c}} \times 21.7^{\mathrm{c}}$ \\
\hline $\mathrm{I}_{2} \mathrm{C}_{2}$ & $30.8^{\mathrm{d}} \times 17.8^{\mathrm{d}}$ & $31.5^{\mathrm{d}} \times 21.7^{\mathrm{d}}$ \\
\hline $\mathrm{I}_{3} \mathrm{C}_{2}$ & $35.2^{\mathrm{b}} \times 21.7^{\mathrm{b}}$ & $30.8^{\mathrm{d}} \times 16.8^{\mathrm{d}}$ \\
\hline $\mathrm{I}_{1} \mathrm{C}_{3}$ & $35.2^{\mathrm{b}} \times 19.3^{\mathrm{c}}$ & $35.7^{\mathrm{b}} \times 25.2^{\mathrm{b}}$ \\
\hline $\mathrm{I}_{2} \mathrm{C}_{3}$ & $33.8^{\mathrm{c}} \times 19.2^{\mathrm{c}}$ & $33.8^{\mathrm{c}} \times 21.00^{\mathrm{c}}$ \\
\hline $\mathrm{I}_{3} \mathrm{C}_{3}$ & $37.8^{\mathrm{a}} \times 25.20^{\mathrm{a}}$ & $33.8^{\mathrm{c}} \times 19.2^{\mathrm{c}}$ \\
\hline $\mathrm{I}_{1} \mathrm{C}_{4}$ & $37.1^{\mathrm{a}} \times 24.7^{\mathrm{a}}$ & $37.8^{\mathrm{a}} \times 29.4^{\mathrm{a}}$ \\
\hline $\mathrm{I}_{2} \mathrm{C}_{4}$ & $35.4^{\mathrm{a}} \times 24.2^{\mathrm{a}}$ & $35.7^{\mathrm{a}} \times 25.2^{\mathrm{a}}$ \\
\hline $\mathrm{I}_{3} \mathrm{C}_{4}$ & $<0.0001(\mathrm{~L})$ & $35.7^{\mathrm{a}} \times 21.7^{\mathrm{a}}$ \\
\hline $\mathrm{Pr}_{2} \mathrm{~F}$ & $<0.0001(\mathrm{~W})$ & $<0.0001(\mathrm{~L})$ \\
\hline $\mathrm{I}_{2}$ & $<0.0001(\mathrm{~W})$ \\
\hline
\end{tabular}

I0-No irrigation, I1-100\% ETc, I2-75\% ETc, I3—50\% ETc, C1—Flowering and fruit set (April-May), C2—Fruit growth stage (June-July), C3-Pre-harvest stage (August-15 September), C4-throughout the growing season (April-September).

\subsection{Leaf Nutrient Status}

From the results, it is pertinent that plants exposed to the $100 \%$ and $75 \%$ ETc treatments had significantly higher concentrations of nutrients $(\mathrm{N}, \mathrm{P}$, and $\mathrm{K})$ in their leaf tissue 
compared to all other treatments (Table 8). The highest leaf $\mathrm{N}$ content (2.03\%), P content $(0.28 \%)$, and $\mathrm{K}$ content $(1.35 \%)$ were recorded at 100 percent ETc $\left(\mathrm{I}_{1}\right)$ level of irrigation which was significantly at par with leaf $\mathrm{N}$ and $\mathrm{P}$ content at 75 percent ETc level of irrigation. Leaf N, P, and K were 15, 28, and 10 percent higher, respectively, in plants irrigated at $100 \%$ ETc compared to rainfed conditions. Similarly, plants irrigated throughout the growing season $\left(\mathrm{C}_{4}\right)$ stage registered the highest leaf nutrient content. Irrigating plants during the flowering and fruit set stage and fruit growth stage produced similar results with respect to the leaf nutrient content. The $\mathrm{Ca}$ and $\mathrm{Mg}$ concentrations in leaf tissue were also higher under $100 \%$ ET and $75 \%$ ET treatments compared to the rainfed treatment (Table 8). Ca and $\mathrm{Mg}$ content in leaves from fully irrigated plants was 11 and 15 percent higher compared to rainfed conditions and was 12 and 20 percent higher in plants irrigated throughout the growing season $\left(\mathrm{C}_{4}\right.$ stage $)$ compared to plants irrigated only during the pre-harvest stage.

Table 8. Effect of different irrigation levels at various crop phenological stages on leaf nutrient content.

\begin{tabular}{|c|c|c|c|c|c|}
\hline Irrigation Level (I) & $\mathbf{N}$ & $\mathbf{P}$ & $\mathbf{K}$ & $\mathrm{Ca}$ & Mg \\
\hline $\mathrm{I}_{0}$ & $1.71^{\mathrm{b}}$ & $0.20^{b}$ & $1.21^{\mathrm{b}}$ & $1.28^{b}$ & $0.28^{b}$ \\
\hline $\mathrm{I}_{1}$ & $2.03^{a}$ & $0.28^{a}$ & $1.35^{\mathrm{a}}$ & $1.45^{\mathrm{a}}$ & $0.33^{\mathrm{a}}$ \\
\hline $\mathrm{I}_{2}$ & $1.94^{\mathrm{a}}$ & $0.26^{\mathrm{a}}$ & $1.33^{\mathrm{ab}}$ & $1.41^{\mathrm{a}}$ & $0.31^{\mathrm{a}}$ \\
\hline $\mathrm{I}_{3}$ & $1.87^{\mathrm{ab}}$ & $0.26^{\mathrm{a}}$ & $1.31^{\mathrm{ab}}$ & $1.40^{\mathrm{a}}$ & $0.30^{\mathrm{a}}$ \\
\hline $\operatorname{Pr}>\mathrm{F}$ & 0.012 & 0.004 & 0.0011 & $<0.0001$ & 0.0003 \\
\hline \multicolumn{6}{|c|}{ Crop Phenological Stage (C) } \\
\hline $\mathrm{C}_{1}$ & $1.81^{\mathrm{ab}}$ & $0.25^{b}$ & $1.30^{b}$ & $1.40^{\mathrm{c}}$ & $0.32^{b}$ \\
\hline $\mathrm{C}_{2}$ & $1.85^{\mathrm{ab}}$ & $0.27^{b}$ & $1.32^{b}$ & $1.44^{b}$ & $0.32^{b}$ \\
\hline $\mathrm{C}_{3}$ & $1.76^{b}$ & $0.23^{c}$ & $1.26^{\mathrm{c}}$ & $1.33^{\mathrm{d}}$ & $0.27^{b}$ \\
\hline $\mathrm{C}_{4}$ & $2.34^{\mathrm{a}}$ & $0.31^{\mathrm{a}}$ & $1.45^{\mathrm{a}}$ & $1.51^{\mathrm{a}}$ & $0.34^{\mathrm{a}}$ \\
\hline $\operatorname{Pr}>F$ & $<0.0001$ & $<0.0001$ & $<0.0001$ & $<0.0001$ & 0.0002 \\
\hline \multicolumn{6}{|c|}{ Irrigation Level × Crop Phenological Stage (IC) } \\
\hline $\mathrm{I}_{0}$ & $1.71^{\mathrm{c}}$ & $0.20^{\mathrm{e}}$ & $1.21^{\mathrm{e}}$ & $1.28^{\mathrm{f}}$ & $0.25^{\mathrm{d}}$ \\
\hline $\mathrm{I}_{1} \mathrm{C}_{1}$ & $1.92^{b}$ & $0.26^{\mathrm{abcd}}$ & $1.32^{\mathrm{cd}}$ & $1.43^{\mathrm{cd}}$ & $0.34^{b c}$ \\
\hline $\mathrm{I}_{2} \mathrm{C}_{1}$ & $1.88^{b}$ & $0.25^{\text {bcde }}$ & 1.30 cde & $1.40^{\text {cde }}$ & $0.32^{c d}$ \\
\hline $\mathrm{I}_{3} \mathrm{C}_{1}$ & $1.77 \mathrm{bc}$ & 0.25 cde & 1.29 de & 1.38 de & $0.32 \mathrm{~cd}$ \\
\hline $\mathrm{I}_{1} \mathrm{C}_{2}$ & $1.87^{b}$ & $0.28^{a b c d}$ & $1.36^{\mathrm{cd}}$ & $1.46^{\mathrm{ef}}$ & $0.33^{b c}$ \\
\hline $\mathrm{I}_{2} \mathrm{C}_{2}$ & $1.81^{b c}$ & $0.27^{\mathrm{abcd}}$ & $1.33^{\mathrm{cd}}$ & $1.43^{\mathrm{ef}}$ & $0.32^{b c}$ \\
\hline $\mathrm{I}_{3} \mathrm{C}_{2}$ & $1.75^{c}$ & 0.24 cde & $1.27 \mathrm{de}$ & $1.43^{\mathrm{f}}$ & $0.30^{c d}$ \\
\hline $\mathrm{I}_{1} \mathrm{C}_{3}$ & $1.78^{b c}$ & 0.24 cde & $1.27 \mathrm{de}$ & $1.34^{b c}$ & $0.29 \mathrm{bc}$ \\
\hline $\mathrm{I}_{2} \mathrm{C}_{3}$ & $1 . .76^{b c}$ & $0.23^{\text {de }}$ & $1.26 \mathrm{de}$ & $1.34^{\mathrm{cd}}$ & $0.28^{b c}$ \\
\hline $\mathrm{I}_{3} \mathrm{C}_{3}$ & $1.75^{b c}$ & $0.23^{\mathrm{de}}$ & $1.26^{\mathrm{de}}$ & $1.31^{\mathrm{cd}}$ & $0.27^{b c}$ \\
\hline $\mathrm{I}_{1} \mathrm{C}_{4}$ & $2.48^{\mathrm{a}}$ & $0.31^{a}$ & $1.48^{\mathrm{a}}$ & $1.58^{\mathrm{a}}$ & $0.36^{\mathrm{a}}$ \\
\hline $\mathrm{I}_{2} \mathrm{C}_{4}$ & $2.35^{\mathrm{a}}$ & $0.31^{a b}$ & $1.46^{b}$ & $1.51^{b}$ & $0.34^{\mathrm{ab}}$ \\
\hline $\mathrm{I}_{3} \mathrm{C}_{4}$ & $2.19^{a b}$ & $0.30^{a b c}$ & $1.42^{b c}$ & $1.45^{b c}$ & $0.33^{a b c}$ \\
\hline $\operatorname{Pr}>F$ & 0.0001 & $<0.0001$ & $<0.0001$ & $<0.0001$ & 0.008 \\
\hline
\end{tabular}

\subsection{Expression of Nutrient Uptake Genes}

Relative expression studies for POTT (potassium transporter) gene to different levels of irrigation at various crop phenological stages showed significant variability. The expression level ranged from 60 to 100\% (where 100\% was kept for positive calibrator) (Figures 8 and 9). 


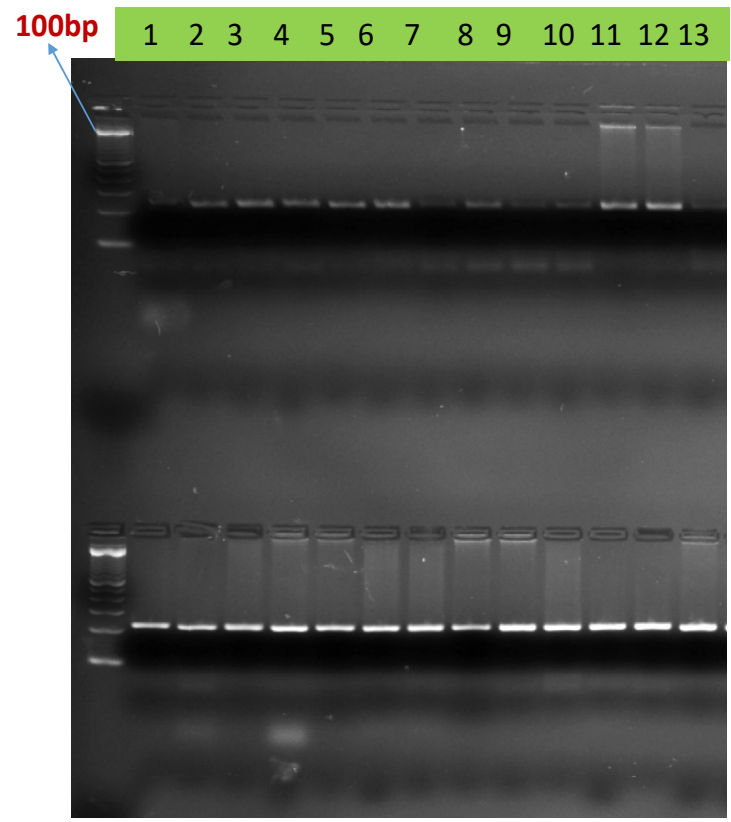

Agrose Gel electrophoresis of gene POTT $(b p=277)$ with Tubulin as control.

Ladder: 100bp DNA ladder
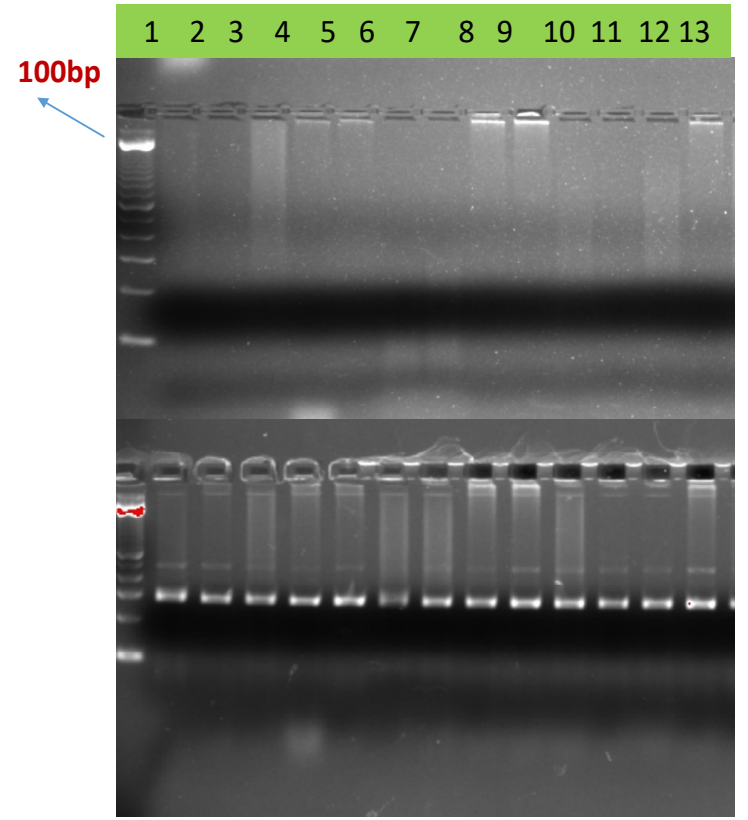

Agrose Gel electrophoresis of gene MdNPFr6.5 (bp=170) with Tubulin as

control.

\begin{tabular}{|c|c|}
\hline 1 & $I_{0}$ \\
\hline 2 & $I_{1} C_{1}$ \\
\hline 3 & $I_{2} C_{1}$ \\
\hline 4 & $I_{3} C_{1}$ \\
\hline 5 & $I_{1} C_{2}$ \\
\hline 6 & $I_{2} C_{2}$ \\
\hline 7 & $I_{3} C_{2}$ \\
\hline 8 & $I_{1} C_{3}$ \\
\hline 9 & $I_{2} C_{3}$ \\
\hline 10 & $I_{3} C_{3}$ \\
\hline 11 & $I_{1} C_{4}$ \\
\hline 12 & $I_{2} C_{4}$ \\
\hline 13 & $I_{3} C_{4}$ \\
\hline
\end{tabular}

Figure 8. Semiquantitative gene expression through Rt-PCR of gene POTT and MdNPFr6.5.

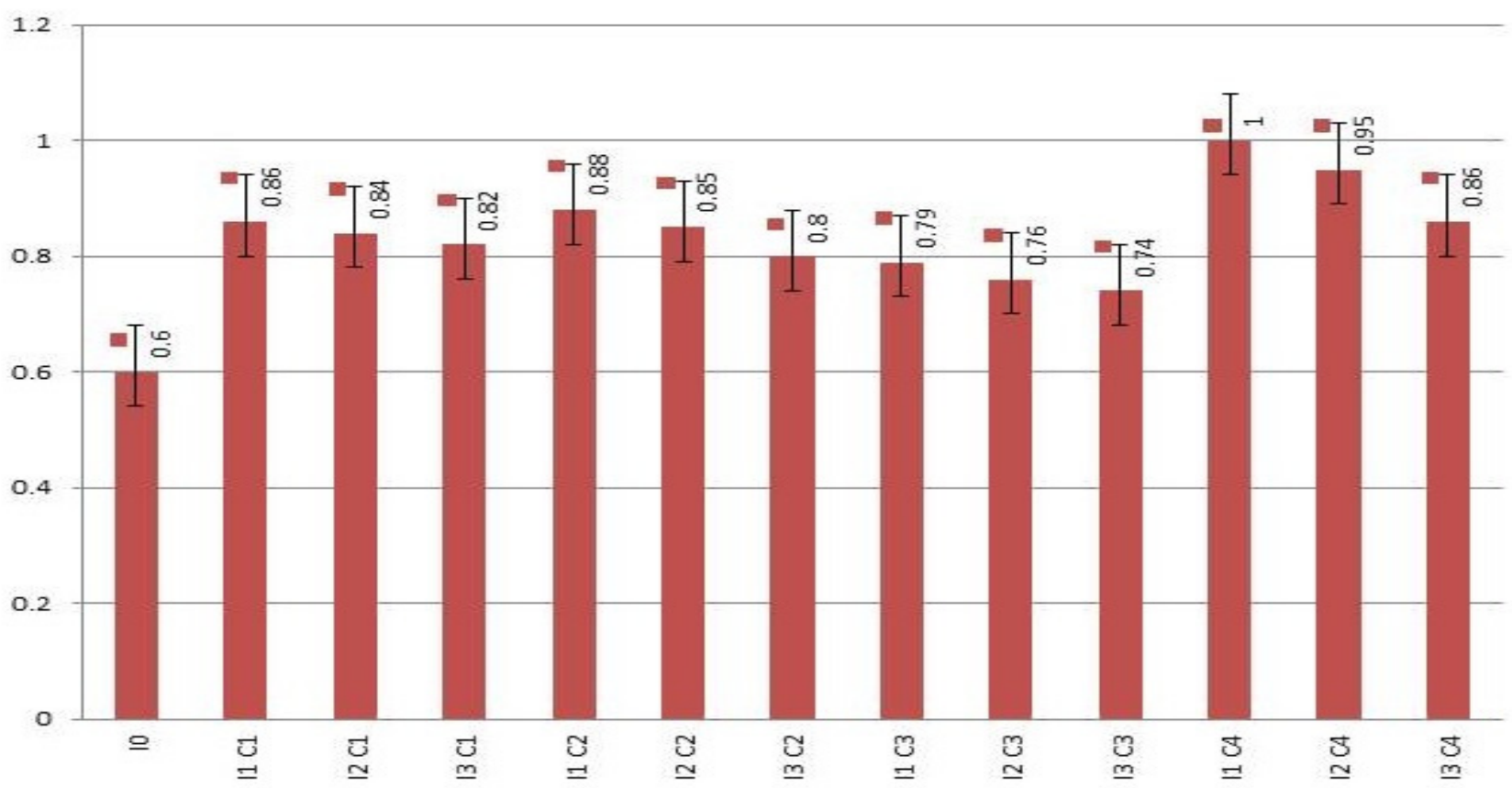

Figure 9. Relative quantification of gene POTT and tubulin in different irrigation levels at various crop phenological stages in apple (cv. Super Chief Sandidge).

\section{Discussion}

The rate of leaf development plays a significant role in fruit productivity of the crop as absorption of photosynthetically active radiations (PAR) and dry matter accumulation primarily depends on the area of leaf and therefore it is imperative to study the influence of irrigation levels on leaf development. The larger the leaf area, the more the PAR is absorbed by the plant and, thus, more accumulation of dry matter. The leaf area under the present 
investigation significantly increased with increasing irrigation, which may be attributed to more frequent callus tissue formation on the well-watered plants. Our findings are similar to those reported by Gigova et al. [25] who advocated that water is the most limiting factor affecting proper leaf area development. Kucukyumuk and Kacal [26] also documented that different irrigation regimes had a significant influence on the leaf area of apples and recorded the highest leaf area in plants frequently irrigated than those irrigated at longer intervals. Similarly, Yuste et al. [27] in grapes, Klamkowski and Treder [28] in strawberry, and Eid et al. [29] in apricot obtained results of a similar trend.

Chlorophyll content in leaves is an important trait for crop production. The corresponding increase in leaf chlorophyll content in plants irrigated at different levels of irrigation at various crop phenological stages was noted to vary between 10 and 31 percent compared to rainfed conditions. Low chlorophyll content in leaves from plants supplied with no irrigation might be due to inhibition of chlorophyll synthesis or disorganization of chloroplasts in the leaves which resulted from water restriction conditions. It might also be due to the significant decrease in mineral contents of leaves, particularly $\mathrm{Mg}$, as $\mathrm{Mg}$ is an important constituent of chlorophyll. In the study, leaves from fully irrigated trees had a higher level of $\mathrm{Mg}$ content that might have accounted for the higher accumulation of chlorophyll content in leaves from fully irrigated plants. Similar observations were made by Trigo-Córdoba et al. [30] who reported low chlorophyll content in grape cultivars under rainfed conditions and 50\% ETc irrigation. Javadi et al. [31] in pear, Haifeng et al. [32] on citrus, and Gholami et al. [33] in fig also documented higher chlorophyll content under irrigated conditions compared to no irrigation.

Leaf relative water content that signifies the metabolic activity in tissues [34] indicating the balance between water absorbed and transpired by leaves, declined significantly due to water restriction conditions under lower levels of ETc and rainfed conditions. This decrease in leaf relative water content could have been due to the unavailability of water in the soil and the leaves could not compensate for water lost through transpiration resulting in low water content [35]. Lower relative water content in plants irrigated only during the flowering and fruit set stage might be due to the reason that leaves during the early season are thin and transpire more water than later in the season. Romero et al. [36] in almond, Satisha et al. [37] in grapes, and Alejandro et al. [38] in apricot also achieved comparable findings in this concern. Stomatal features are known to affect transpiration and, thus, play a vital role in maintaining the water status of plants. The stomata studied were hypostomatic in apple leaves. Kucukyumuk and Kacal [26] also found that stomas are on the lower epidermis of the apple leaves. Reduction in stomatal density and size of leaves under rainfed conditions might be a response to reduced water loss and cell division under water stress conditions. Carbon assimilation of plants relies on the absorption of $\mathrm{CO}_{2}$ by stomata, which partially closes to respond to water deficits. Our findings were again in uniformity with Elias [39] who recorded stomatal closure at periods of strong evaporative demand, which suggested that stomatal density appeared to be primarily influenced by tree water status during the vegetative period in apples. Kour and Bakshi [40] observed that stomatal aperture and density in leaves from peach seedlings significantly change in response to changing water conditions. Previous reports by Basiouny [41] in peach, Misirlı and Aksoy [42] in figs, and Klamkowski and Treder [43] and Kawchaya [44] in strawberry also illustrated a decrease in stomatal density and size due to water stress.

Plant nutrient uptake and subsequent concentrations in various plant parts are influenced by numerous factors including the water status of soil and plant. Water is essential for nutrient uptake by root interception, mass flow, and diffusion. The possible reason for higher nutrient content in leaves of plants subjected to continuous irrigation with drip method may be due to high soil moisture, resulting in the transfer of mineral nutrients and the mass flow of soil solution powered by water absorption and plant root diffusion. The further movement between the soil particles and the rise in mass flow due to a higher transpiration rate as a result of stomata opening improves the transport of nutrients under high soil moisture. Another potential reason for a higher content of leaf nutrients at 100\% 
ETc irrigation levels may be attributed to an extended, more fibrous, and more productive root system, influenced by comparatively improved moisture and thermal regimes, which increased root growth and thus increased the capacity for higher absorption of the nutrient. Less water availability under water stress conditions generally results in a reduction in total nutrient uptake and subsequently reduces the concentration of mineral nutrients in plants [45].

Ca and Mg uptake is primarily through mass flow [10], therefore the plants irrigated at 100\% and $75 \%$ ETc had the highest $\mathrm{Ca}$ and $\mathrm{Mg}$ concentrations in their leaves. It is because roots absorb more nutrients, especially calcium and magnesium, from the moisture-rich soil compared to dry soil as a result of more extensive root growth [46]. Furthermore, transpiration rate has important significance for the transport of nutrients from the soil to the top of the plant, and this rate has particular significance for xylem mobile nutrients such as $\mathrm{Ca}$; the decrease in transpiration rate as a consequence of water stress under rainfed conditions might have decreased the uptake of these mobile nutrients. Kacar and Katkat [47] also have stated that uptake of nutrients increased with transpiration increasing factors. Thaur et al. [48] reported that drip irrigation at 100\% ETc significantly increased leaf nitrogen $(\mathrm{N})$, phosphorus $(\mathrm{P})$, and potassium $(\mathrm{K})$ in apple trees planted under high density plantation. In general, Shirgure et al. [49] in acid lime, Chauhan and Chandel [50] in kiwifruit, Küçükyumuk et al. [51] in cherry, and Zhong et al. [52] in apple also documented similar results. The variation in gene expression of potassium transporter gene was found to be due to variation in irrigation levels at various crop phenological stages. Variation in transporter genes was also found in accordance with the change in fruit size. Therefore, the availability of water significantly influences gene expression. Similar kinds of variation in nutrient transporter genes have been reported by Dechorgnat et al. [53], who found that expression of nutrient transporter genes was regulated by nutrient availability and which in turn depends on the availability of water. Therefore, water availability significantly influences nutrient uptake by regulating transporter genes. Similarly, nitrogen inducible transporter showed a strong expression in guard cells and supports the stomatal function in the presence of available forms of nitrogen [54]. Song et al. [55] also found that in response to potassium deficiency expression of the potassium transporter gene, PpeKUP6 was dramatically reduced in peach leaves.

\section{Conclusions}

Leaf physiological characteristics like leaf area, leaf relative water content, and leaf chlorophyll content in apples under high-density plantation grown in medium loam soils under temperate conditions are highly influenced by the proper supply of irrigation using a drip irrigation system, significantly during the early growth stages like flowering and fruit set stage. Irrigation amount likely does not have any influence on leaf development after the fruit growth stage. Stomatal opening and stroma size greatly vary from no irrigation to optimum irrigation in these plants. High density apple trees exposed to optimum irrigation levels $(100 \%$ and $75 \%$ ET) had significantly higher concentrations of nutrients $(\mathrm{N}, \mathrm{P}$, and $\mathrm{K})$ in their leaf tissues. The concentration of $\mathrm{Ca}$ and $\mathrm{Mg}$ nutrients in leaf tissues are greatly influenced by the optimum supply of water during the early stages of apple growth. It is therefore observed that stage $C_{1}$ and stage $C_{2}$ are the critical growth stages of apple for optimum leaf development and proper nutrient uptake. Since irrigation regimes were found to have a differential influence on the expression of different genes/pathways and hence can affect the metabolism of apple plants. Therefore, it is confirmed that genes influenced under various irrigation levels are proportionally responsive and effective concerning survival and performance. As potassium is important in the photosynthesis and defense of plants, the expression of the potassium transporter gene under higher levels of irrigation will have a positive influence upon the plant survival under both biotic and abiotic stress. This study can pave way for further research in the expression of transport genes since not many advanced findings have been performed in this field. 
Author Contributions: Conceptualization, M.K.S. and R.M.; methodology, J.I.M.; software, J.I.M.; validation, K.M., A.R.M., and S.A.B.; formal analysis, R.M., S.M.; investigation, R.M. resources J.I.M. and S.A.B.; writing-R.M., S.M.P., D.I.H., S.M., S.N. and H.A.E.-S.; writing-review and editing D.I.H. and R.M. All authors have read and agreed to the published version of the manuscript.

Funding: This research was funded by SERB-CII, DST, Govt of India in collaboration with Jain Irrigation System Limited as a fellowship grant to Rafiya Mushtaq, (SERB/PM Fellow/CII-FICCI/2018).

Institutional Review Board Statement: Not applicable.

Informed Consent Statement: Not applicable.

Data Availability Statement: Not applicable.

Acknowledgments: Rafiya Mushtaq is highly grateful to SERB-CII, DST, India for selecting her for India's prestigious fellowship "Prime Ministers Fellowship for Doctoral Research". She is also thankful to Jain Irrigation System Limited for supporting her fellowship. The authors are grateful ICAR-CITH Lab and K-Lab SKUAST-K for providing necessary lab facilities. The authors would like to extend their sincere appreciation to the Researchers Supporting Project Number (RSP-2021/19), King Saud University, Riyadh, Saudi Arabia.

Conflicts of Interest: The authors declare no conflict of interest.

\section{References}

1. Seckler, D.; Amarasinghe, U.; Molden, D.; de Silva, R.; Barker, R. World Water Demand and Supply, 1990 to 2025: Scenarios and Issues; IWMI Research Report 19; IWMI: Colombo, Sri Lanka, 1998.

2. Blanco, V.; Martínez-Hernández, G.B.; Artés-Hernández, F.; Blaya-Ros, P.J.; Torres-Sánchez, R.; Domingo, R. Water relations and quality changes throughout fruit development and shelf life of sweet cherry grown under regulated deficit irrigation. Agric. Water Manag. 2019, 217, 243-254. [CrossRef]

3. Blanco, V.; Blaya-Ros, P.J.; Torres-Sánchez, R.; Domingo, R. Influence of Regulated Deficit Irrigation and Environmental Conditions on Reproductive Response of Sweet Cherry Trees. Plants 2020, 9, 94. [CrossRef]

4. Zanotelli, D.; Montagnani, L.; Andreotti, C.; Tagliavini, M. Evapotranspiration and crop coefficient patterns of an apple orchard in a sub-humid environment. Agric. Water Manag. 2019, 226, 105756. [CrossRef]

5. Fallahi, E. Influence of Rootstock and Irrigation Methods on Water Use, Mineral Nutrition, Growth, Fruit Yield, and Quality in 'Gala' Apple. HortTechnology 2012, 22, 731-737. [CrossRef]

6. Ozturk, Z.N.; Talamè, V.; Deyholos, M.; Michalowski, C.B.; Galbraith, D.W.; Gozukirmizi, N.; Tuberosa, R.; Bohnert, H.J. Monitoring large-scale changes in transcript abundance in drought- and salt-stressed barley. Plant Mol. Biol. 2002, 48, 551-573. [CrossRef]

7. Peter, F.; Waterman, P.A. Fertigation Guidelines in High Density Apples and Apple Nurseries in the Okanagan-Similkameen; Ministry of Agriculture, Food and Fisheries: Vancouver, BC, Canada, 2001.

8. Fallahi, E.; Fallahi, B.; Shafii, B.; Morales, B. Water use, tree growth and leaf mineral nutrients of young 'Fuji' apples as influenced by different irrigation systems. In Proceedings of the International Symposium on Mineral Nutrition of Fruit Plants, Talca, Chile, 31 October 2006.

9. Fallahi, E.; Fallahi, B.; Neilsen, G.H.; Neilsen, D.; Peryea, F.J. Effects of mineral nutrition on fruit quality and nutritional disorders in apples. Acta Hortic. 2010, 868, 49-60. [CrossRef]

10. Zegbe, J.A.; Serna-Pérez, A.; González-Fuentes, J.A. Nutrient status of apple leaves not affected by three years of irrigation using partial rootzone drying. J. Plant Nutr. Soil Sci. 2011, 174, 459-464. [CrossRef]

11. Neilsen, G.H.; Neilsen, D. Nutritional requirements of apple. In Apples: Botany, Production and Uses; Ferree, D.C., Warrington, I.J., Eds.; CABI Publishing: Cambridge, MA, USA, 2003; pp. 267-302.

12. Grabov, A. Plant KT/KUP/HAK Potassium Transporters: Single Family-Multiple Functions. Ann. Bot. 2007, 99, $1035-1041$. [CrossRef]

13. Zhao, D.; Oosterhuis, D.M.; Bednarz, C.W. Influence of Potassium Deficiency on Photosynthesis, Chlorophyll Content, and Chloroplast Ultrastructure of Cotton Plants. Photosynthetica 2001, 39, 103-109. [CrossRef]

14. Ashley, M.K.; Grant, M.; Grabov, A. Plant responses to potassium deficiencies: A role for potassium transport proteins. J. Exp. Bot. 2006, 57, 425-436. [CrossRef]

15. Song, Z.Z.; Su, Y.H. Distinctive potassium-accumulation capability of alligator weed (Alternanthera philoxeroides) links to highaffinity potassium transport facilitated by K+ uptake systems. Weed Sci. 2013, 61, 77-84. [CrossRef]

16. Song, Z.Z.; Yang, S.Y.; Zhu, H.; Jin, M.; Su, Y.H. Heterologous expression of an alligator weed high-affinity potassium transporter gene enhances salinity tolerance in Arabidopsis. Am. J. Bot. 2014, 101, 840-850. [CrossRef] [PubMed]

17. Piper, C.S. Soil and Plant Analysis; Hans Publication: Bombay, India, 1966; p. 164.

18. Arnon, Z.I. Determination of chlorophyll. Plant Physiol. 1949, 24, 1-15. [CrossRef] [PubMed] 
19. Richardson, A.D.; Duigan, S.P.; Berlyn, G.P. An evaluation of noninvasive methods to estimate foliar chlorophyll content. New Phytol. 2002, 153, 185-194. [CrossRef]

20. Slavik, B. Methods of Studying Plant Water Relations; Springer: Berlin/Heidelberg, Germany, 1974; Volume XVIII, p. 452.

21. Beakbane, A.B.; Majumdar, P.K. A relationship between stomatal density and growth potential in apple rootstocks. J. Hortic. Sci. 1975, 50, 285-289. [CrossRef]

22. Chapman, H.D. Suggested foliar sampling and handling techniques for determining the nutrient status of some field, horticultural and plantation crops. Indian J. Hortic. 1964, 21, 97-117.

23. Jackson, M.L. Soil Chemical Analysis; Asia Publishing House: Bombay, India, 1967.

24. Livak, K.J.; Schmittgen, T.D. Analysis of relative gene expression data using real-time quantitative PCR and the 2(-Delta Delta $\mathrm{C}(\mathrm{T})$ ) method. Methods 2001, 25, 402-408. [CrossRef] [PubMed]

25. Gigova, A.; Moteva, M.; Mitova, T.; Kostadinov, G. Irrigation impact on leaf area and net photosynthetic productivity of Canola. In Proceedings of the 5th International Conference on Trends in Agricultural Engineering (TEA-2013), Prague, Czech Republic, 3-6 September 2013; pp. 173-180.

26. Kucukyumuk, C.; Kacal, E. The Effects of different irrigation programmes in drip irrigation on leaf properties of Starkrimson delicious apple variety. Bull. UASVM Hortic. 2010, 67, 297-300.

27. Yuste, J.; Rubioy, J.A.; Perez, M.A. Influence of plant density and water regime on soil water use, water relations and productivity of trellis trained tempranillo grape vines. Acta Hortic. 2004, 646, 187-193. [CrossRef]

28. Klamkowski, K.; Treder, W. Response to drought stress of three strawberry cultivars grown under greenhouse conditions. J. Fruit Ornam. Plant Res. 2008, 16, 179-188.

29. Eid, T.A.; Fatama, I.; Grah, A.; Hussein, S.M. Effect of soil moisture regimes and potassium application on growth, yield and fruit quality of "Canino" apricot (Prunus armeniaca L.). J. Plant Prod. 2014, 4, 621-640. [CrossRef]

30. Trigo-Córdoba, E.; Bouzas-Cid, Y.; Orriols-Fernández, I.; Mirás-Avalos, J.M. Effects of deficit irrigation on the performance of grapevine (Vitis vinifera L.) cvs. Godello and Treixadura in Ribeiro, NW Spain. Agric. Water Manag. 2015, 161, 20-30. [CrossRef]

31. Javadi, T.; Arzani, K.; Ebrahimzadeh, H. Study of proline, soluble sugar and chlorophyll A and B changes in nine Asian and one European pear cultivar under drought stress. Acta Hortic. 2008, 769, 241-246. [CrossRef]

32. Haifeng, G.; Cuina, F.; Xinnan, L. Effects of drought stress on antioxidant system of leaves from different citrus rootstocks. J. Agric. Sci. Technol. 2011, 1, 32-35.

33. Gholami, M.; Rahemi, M.; Kholdebarin, B.; Rastegar, S. Biochemical responses in leaves of four fig cultivars subjected to water stress and recovery. Sci. Hortic. 2012, 148, 109-117. [CrossRef]

34. Flower, D.J.; Ludlow, M.M. Contribution of osmotic adjustment to the dehydration tolerance of water stressed pigeon pea (Cajanas cajan (L) Milsp) leaves. Plant Cell Environ. 1986, 9, 33-40.

35. Gadallah, M.A.A. Effects of indole-3-acetic acid and zinc on the growth, osmotic potential and soluble carbon and nitrogen components of soybean plants growing under water deficit. J. Arid. Environ. 2000, 44, 451-467. [CrossRef]

36. Romero, P.; Botia, P.; Garcia, F. Effects of regulated deficit irrigation under subsurface drip irrigation conditions on vegetative development and yield of mature almond trees. Plant Soil 2004, 260, 169-181. [CrossRef]

37. Satisha, J.; Prakash, G.S.; Bhatt, R.M.; Sampathkumar, P. Effect of soil moisture stress on physiological response in grape (Vitis vinifera L.) varieties. J. Hortic. Sci. 2006, 1, 99-103.

38. Alejendro, P.P.; Domingo, R.; Torrecillas, A.; Ruiz-Sanchez, M.C. Response of apricot trees to deficit irrigation strategies. Irrig. Sci. 2009, 27, 231.

39. Elias, P. Stomata density and size of apple trees growing in irrigated and non-irrigated conditions. Biol. Bratisl. 1995, 50, 115-118.

40. Kour, G.; Bakshi, P. Irrigation Management Practices and Their Influence on Fruit Agroecosystem. In Irrigation in Agroecosystems; BoD—Books on Demand: Norderstedt, Germany, 2018; pp. 21-36. [CrossRef]

41. Basiouny, F.M. Response of peach seedlings to water stress and saturation conditions. Proc. Fla. State Hortic. Soc. 1977, 90, 261-263.

42. Misırlı, A.; Aksoy, U. The researches on leaf properties and stoma distrubition of Sarılop fig clone. Ege Univ. J. Agric. Fac. 1994, 31, 57-63.

43. Klamkowski, K.; Treder, W. Morphological and physiological response of strawberry plants to water stress. Agric. Conspec. Sci. 2006, 71, 159-165.

44. Kachwaya, D.S. Studies on Drip Irrigation and Fertigation in Strawberry (Fragaria $\times$ ananassa Duch.). Ph.D. Thesis, Dr Yashwant Singh Parmar University of Horticulture and Forestry, Nauni, India, 2014.

45. Garg, B.K. Nutrient uptake and management under drought: Nutrient-moisture interaction. Curr. Agric. 2003, 27, 1-8.

46. Havlin, J.L.; Beaton, J.D.; Tisdale, S.L.; Nelson, W.L. Soil Fertility and Fertilizers, an Introduction to Nutrient Management, 6th ed.; Macmillan, Inc.: New York, NY, USA, 1999; p. 409.

47. Kacar, B.; Katkat, A.V. Gübreler ve Gübreleme Tekniği, 2nd ed.; Nobel Yayin No: 1119; Nobel Basımevi, 560 s; Fen ve Biyoloji Yayınları Dizisi: Ankara, Turkey, 2007.

48. Thakur, J.; Sharma, J.C.; Mohit, S.; Verma, P. Vegetative Growth and Foliage Nutrient Content of Super Chief Apple under Different Irrigation and Fertigation Schedules in NW Himalayan Region. Commun. Soil Sci. Plant Anal. 2020, 51, $2174-2188$. [CrossRef] 
49. Shirgure, P.S.; Srivastava, A.K.; Singh, S.; Pimpale, A.R. Drip irrigation scheduling, growth, yield and quality of acid Lime (Citrus aurantifolia). Indian J. Agric. Sci. 2004, 74, 92-94.

50. Chauhan, N.; Chandel, J.S. Growth, productivity, leaf nutrient contents and water-use efficiency of kiwifruit (Actinidia deliciosa) under drip and basin irrigation system. Indian J. Agric. Sci. 2010, 80, 584-587.

51. Küçükyumuk, Z.; Küçükyumuk, C.; İbrahim, E.I.; Eraslan, F. Effects of different sweet cherry rootstocks and drought stress on nutrient concentrations. J. Agric. Sci. 2015, 21, 431-438. [CrossRef]

52. Zhong, Y.; Fei, L.; Li, Y.; Zeng, J.; Dai, Z. Response of fruit yield, fruit quality, and water use efficiency to water deficits for apple trees under surge-root irrigation in the loess plateau of China. Agric. Water Manag. 2019, 222, 221-230. [CrossRef]

53. Dechorgnat, K.L.; Francis, K.L.; Dhagga, K.S.; Rafalski, J.A.; Tyermal, S.D.; Kaiser, B.N. Tissue and nitrogen linked expression profiles of ammonium and nitrate transporters in maize. BMC Plant Biol. 2019, 19, 206. [CrossRef] [PubMed]

54. Guo, F.Q.; Young, J.; Crawford, N.M. The nitrate transporter AtNRT1. 1 (CHL1) functions in stomatal opening and contributes to drought susceptibility in Arabidopsis. Plant Cell 2003, 15, 107-111. [CrossRef]

55. Song, Z.Z.; Yang, Y.; Ma, R.J.; Xu, J.L.; Yu, X.L. Transcription of potassium transporter genes of KT/HAK/KUP family in peach seedlings and responses to abiotic stresses. Biol. Plant. 2015, 59, 65-73. [CrossRef] 